\title{
DESIGN, SYNTHESIS, MOLECULAR DOCKING AND BIOLOGICAL EVALUATION OF SOME NOVEL QUINAZOLIN-4(3H)-ONE DERIVATIVES AS ANTI-INFLAMMATORY AGENTS
}

\author{
Mohamed-Kamal Ibrahim ${ }^{\mathrm{a}}$, Khaled El-Adl ${ }^{\mathrm{a}, *}$, Ahmed A. Al-Karmalawy ${ }^{\mathrm{a}}$ \\ ${ }^{a}$ Pharmaceutical Chemistry Department, Faculty of Pharmacy, Al-Azhar University, Cairo, Egypt
}

\begin{abstract}
In view of their expected anti-inflammatory activity, novel series of 6-iodo-2-phenylquinazolin-4(3H)-one (4-12 $2_{\mathrm{a}-\mathrm{d}}$ ) were designed and synthesized in order to evaluate their antiinflammatory activity using carrageenan-induced rat paw edema assay. Most of the novel quinazolinone derivatives showed considerable potent anti-inflammatory activities of superior G.I.T. safety profile in experimental rats in comparing to indomethacin as a reference drug. The molecular docking was performed for all synthesized compounds to assess their binding affinity to COX-2 enzyme in order to rationalize their anti-inflammatory activity in a qualitative way. The obtained data from the molecular modeling was strongly correlated with that obtained from the biological screening. The highest binding affinities were noticed for compounds $\mathbf{8}_{\mathfrak{a}}, \mathbf{1 2}_{\mathbf{b}}$ and $\mathbf{1 0}_{\mathbf{b}}$ which showed the highest anti-inflammatory activities of this series. Compounds $\mathbf{1 2}_{\mathrm{b}}, \mathbf{9}_{\mathrm{c}}$ and $\mathbf{8}_{\mathrm{a}}$ exhibited the least ulcerogenic effect in all of the experimental animals.
\end{abstract}

Keywords: 6-Iodoquinazolin-4(3H)-ones, Molecular docking, COX-2, Anti-inflammatory.

\section{INTRODUCTION}

In recent years there is a tremendous increase of inflammatory cases, leading to the design and development of newer anti-inflammatory agents (Rajasekaran and Gopalkrishna, 2012). It is well known that non steroidal anti-inflammatory drugs (NSAIDs) are associated with several side effects such as gastrointestinal mucosal damage, bleeding, intolerance and renal toxicity. Production of safer and more active NSAIDs and analgesic drugs is still needed (Amin $\boldsymbol{e t}$ al., 2010). 4(3H)-Quinazolinones constitute an important family of compounds in heterocyclic chemistry and exhibit a broad spectrum of medicinal values including anticonvulsant (Kumar $\boldsymbol{e t}$ al., 2011), antiviral (Krishnan et al., 2011), antibacterial (Myangar and Raval, 2012), antifungal (Hassanzadeh et al., 2012), antimalarial (Sen et al., 2010), anticancer (Sirisoma et al., 2010), antihistaminic (Alagarsamy et al., 2009), serotonin 5-HT 2 antagonists (Sati et al., 2009), antiulcer (Patil $\boldsymbol{e t}$ al., 2010), antihyperlipidemic (Refaie $\boldsymbol{e t}$ al., 2005), antihypertensive (Abou-Seri $\boldsymbol{e t}$ al., 2011), anti-inflammatory and analgesic activities (Abbas et al., 2010; Alafeefy et al., 2010; Mohamed et al., 2011; Rajveer et al., 2010). As a result, quinazolinones and their derivatives are regarded as "privileged structures" that are capable of binding to multiple receptors with high affinity (Naganaboina et al., 2011). Additionally, different known anti-inflammatory drugs such as: Proquazone, 1-isopropyl-7-methyl-4-phenylquinazoline-2(1H)-one (1) (Amin et al., 2010), Fluoroquazone, 4-(4-fluorophenyl)-1-isopropyl-7-methylquinazolin-2(1H)-one (2) (Chandrika $\boldsymbol{e t}$ al., 2008) and Tryptanthrin, indolo[2,1-b]quinazoline alkaloid (3) (Figure 1) (Amin et al., 2010) are bearing quinazoline nucleus. The overall anti-inflammatory profile of proquazone is comparable with that of indomethacin (Chandrika et al., 2008). Furthermore, it has been reported that substitution pattern by different aryl or heteroaryl moieties at $2 / 3$ position of quinazolinone nucleus markedly influences its anti-inflammatory activity (Amin et al., 2010; Rajasekaran and Gopalkrishna, 2012). On the other hand, pyrazoles (Mohamed et al., 2011), pyrimidones (Mohamed et al., 2010), pyrimidinethiones (Mohamed et al., 2009), 2-oxo (imino) pyridines (Amin et al., 2010) and pyrans (Mohamed et al., 2011) are other important pharmacodynamic heterocyclic nuclei which when incorporated into different heterocyclic templates, have been 
reported to possess potent anti-inflammatory activity. Moreover, there are many reports in the literature that quinazolinones derivatives reduce the inflammation in different inflammatory disorders by inhibiting the COX-2 enzyme (Amin et al., 2010; Mohamed et al., 2011). The enhanced overall lipophilic characteristics of the target compounds could favor their selectivity towards COX-2 enzyme over COX-1 leading to increase of GIT safety margin (Amin et al., 2010; Mohamed et al., 2011). Based on the above observations, it was of interest to synthesize a novel series of quinazolinone derivatives with structure modifications involving incorporation of the above mentioned heterocyclic moieties at 3rd position and phenyl moiety at 2nd position of quinazolinone moiety as a trial to obtain safer and potent anti-inflammatory agents. The ulcerogenic activity of the tested compounds was determined.

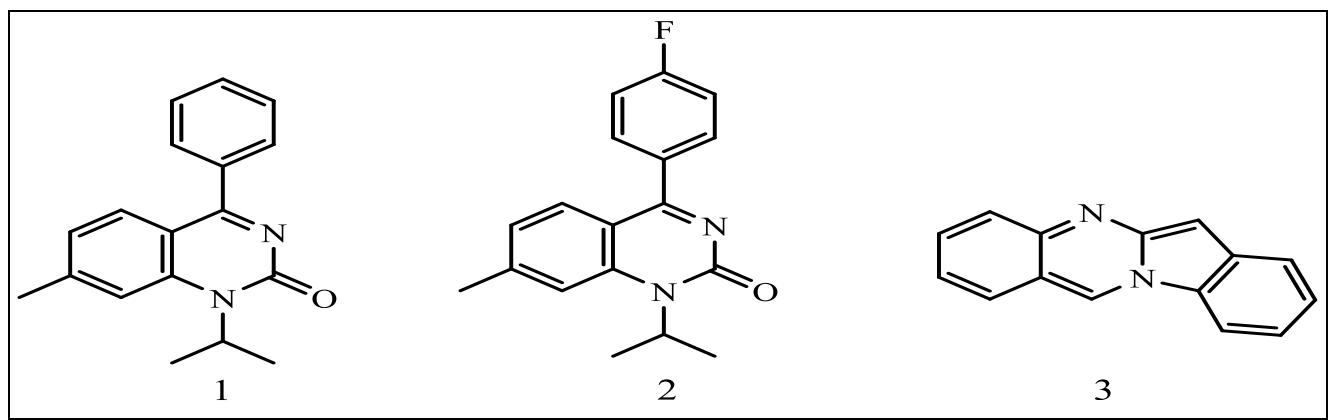

Figure (1): Anti-inflammatory drugs bearing quinazoline nucleus

\section{RESULTS AND DISCUSSION}

\section{A. Chemistry}

The sequence of reactions followed in the syntheses of the target compounds is illustrated in Schemes 1-3. A new series of the title compounds incorporated into diverse $\mathrm{N}$ and $\mathrm{O}$ heterocyclic moieties of pharmacoavailability as anti-inflammatory agents were synthesized starting with anthranilic acid by its reaction with iodine in the presence of aqueous $\mathrm{KOH}$ to give 5iodoanthranilic acid (1) which treated with benzoyl chloride to afford $N$-benzoyl-5-iodoanthranilic acid (2) following the reported procedures (Alafeefy $\boldsymbol{e t}$ al., 2010). Refluxing of $N$-benzoyl-5iodoanthranilic acid (2) in acetic anhydride afforded 6-iodo-2-phenyl-4H-3,1-benzoxazin-4-one (3) which reacted with 4-aminoacetophenone to give the key intermediate compound, 6-iodo-2phenyl-3-(4-acetylphenyl)-4(3H)-quinazolinone (4). Claisen-Schmidt condensation of the acetyl derivative (4) with different aromatic aldehydes afforded the corresponding $\alpha, \beta$-unsaturated ketones (chalcones) $\left(\mathbf{5}_{\mathrm{a}-\mathrm{e}}\right)$ (Scheme 1$)$, which underwent cyclization with hydrazine hydrate in absolute ethanol to afford the corresponding pyrazoline derivatives $\left(\mathbf{6}_{\mathbf{a}-\mathbf{b}}\right)$, but when the reaction was carried out in glacial acetic acid, the $N$-acetyl pyrazoline derivatives (7) were obtained. Also, cyclocondensation of the unsaturated ketones (5) by urea and / or thiourea yielded the corresponding tetrahydropyrimidin-2-ones $\left(\mathbf{8}_{\mathbf{a}-\mathbf{b}}\right)$ and / or tetrahydropyrimidin-2-thiones $\left(\mathbf{9}_{\mathbf{a}-\mathbf{c}}\right)$ respectively. Moreover, cyclocondensation of the unsaturated ketone $\left(\mathbf{5}_{\mathbf{a}}\right)$ by ethyl cyanoacetate afforded 2(1H)-pyridone $\left(\mathbf{1 0}_{\mathbf{a}}\right)$ in $65 \%$ yield (Scheme 2$)$, which was achieved in good yield (80\%) by one pot reaction. The one pot reaction of (4) with the appropriate aromatic aldehydes and either ethyl cyanoacetate or malononitrile in the presence of excess anhydrous ammonium acetate in nbutanol afforded the corresponding 2(1H)-pyridones $\left(\mathbf{1 0}_{\mathrm{a}-\mathrm{e}}\right)$ or $2(1 H)$-iminopyridines (11 $\left.\mathbf{1}_{\mathrm{a}-\mathrm{e}}\right)$ respectively. Upon applying the same procedure, using malononitrile in the presence of piperidine instead of ammonium acetate, the target 2-aminopyrans (12 $\left.\mathbf{a}_{\mathbf{a}-\mathbf{d}}\right)$ were obtained (Scheme 3$)$. 


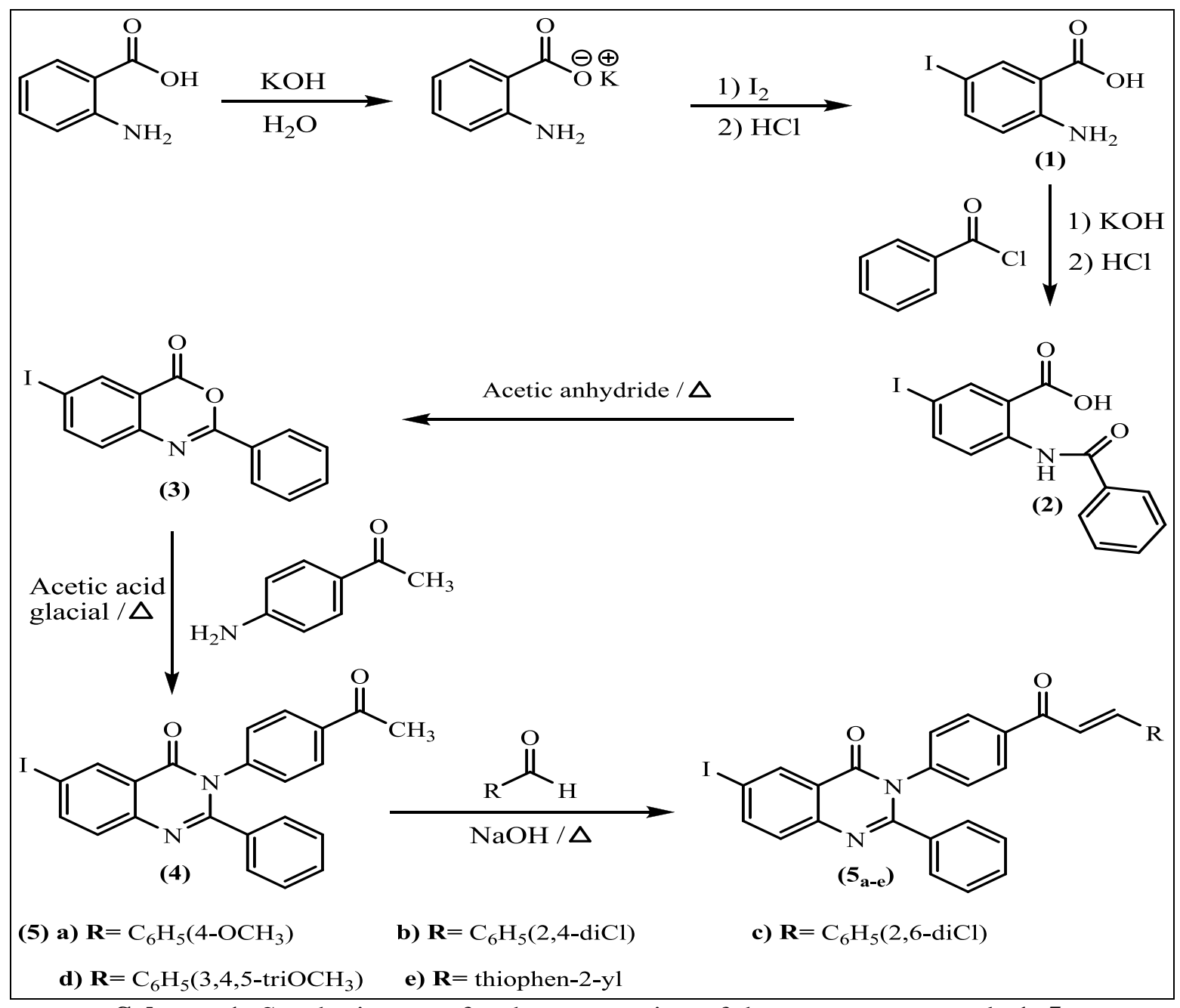

Scheme 1: Synthetic route for the preparation of the target compounds 1-5. 


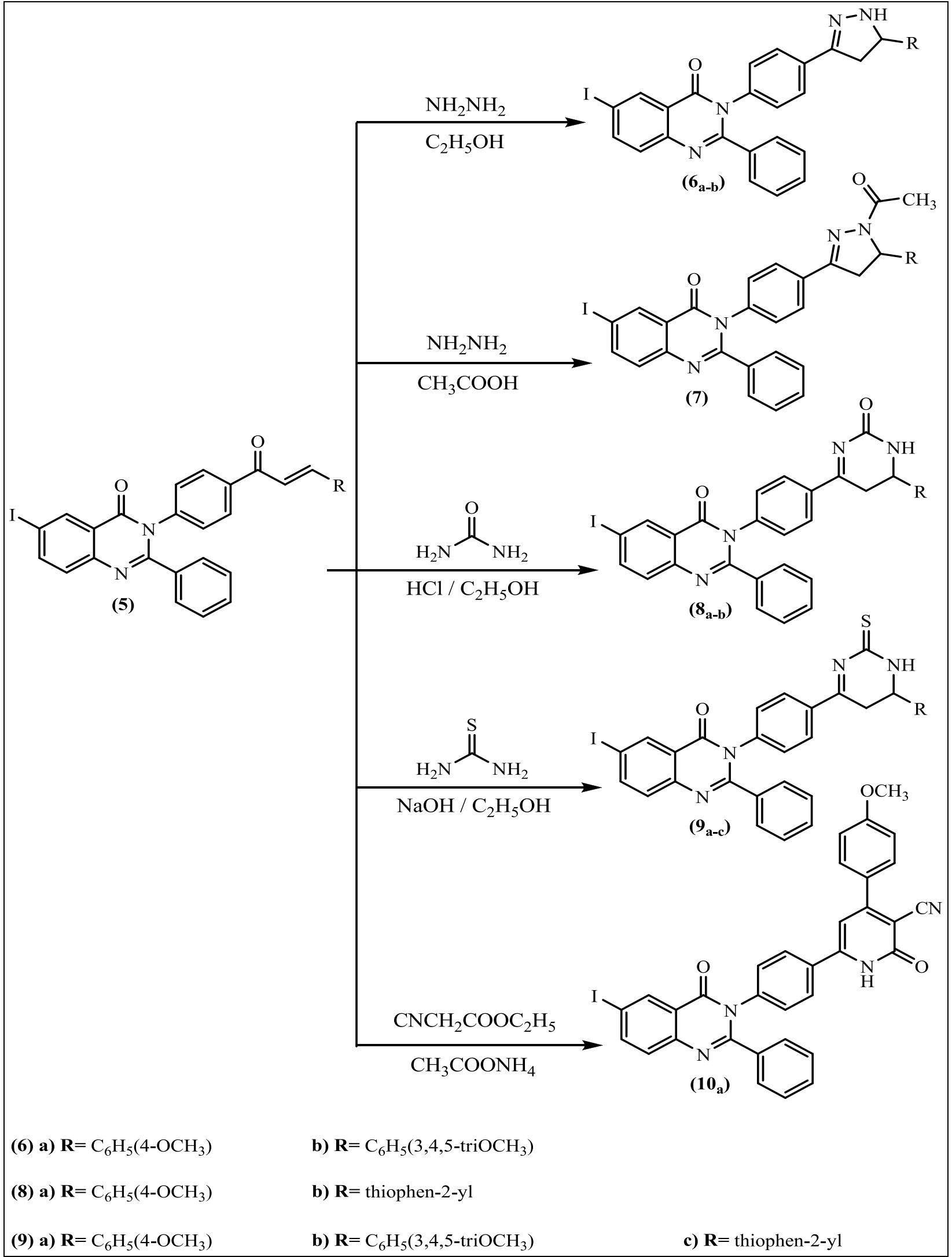

Scheme 2: Synthetic route for the preparation of the target compounds $\mathbf{6}-\mathbf{1 0}_{\mathrm{a}}$. 


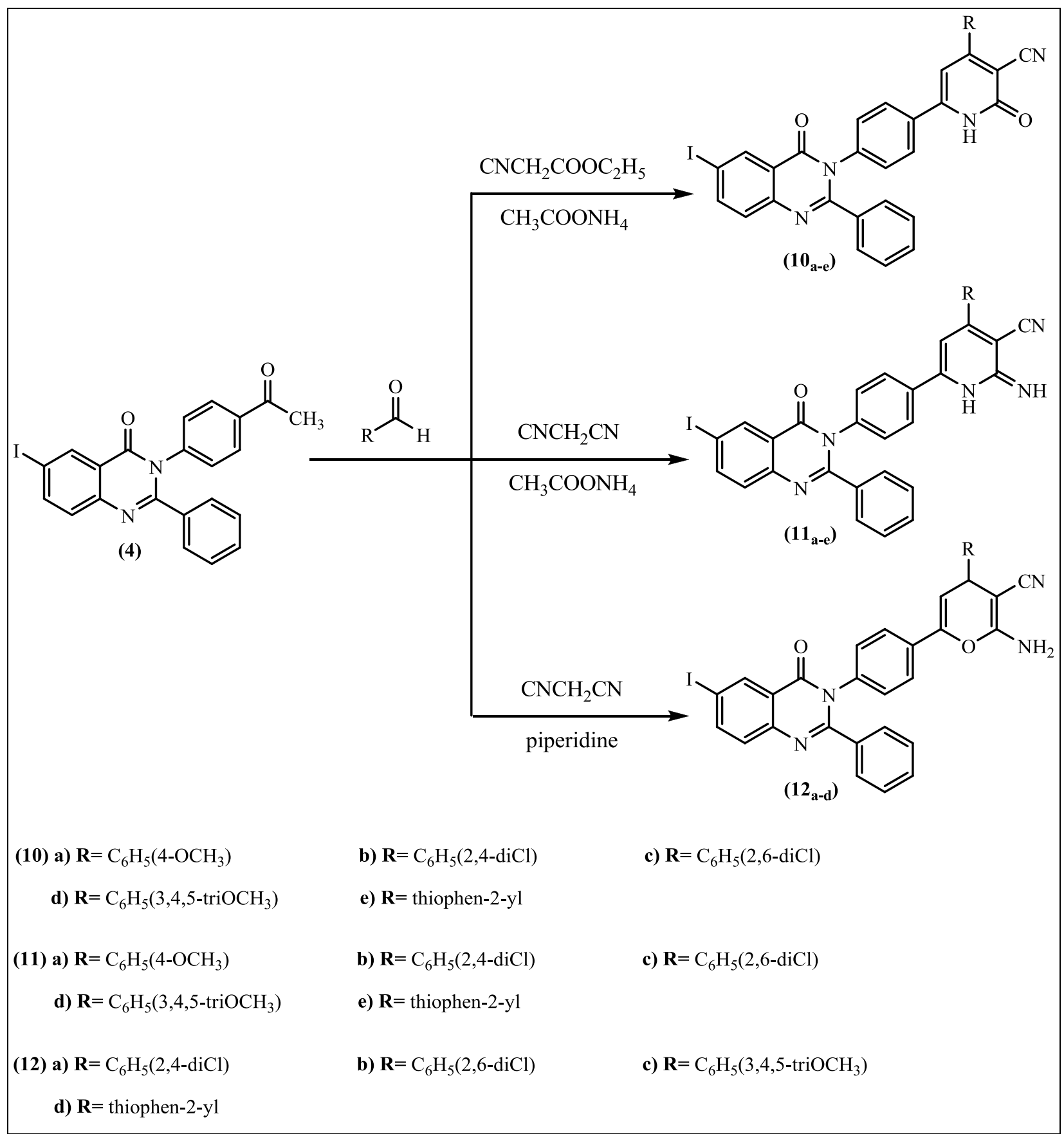

Scheme 3: Synthetic route for the preparation of the target compounds 10-12.

\section{B. Molecular docking study}

The aim of this work was to study the crystal structure of COX-2 and to rationalize the obtained biological data and explain the possible interactions that might take place between the tested derivatives and COX-2 enzyme in comparing to Indomethacin ${ }^{\circledR}$ in order to obtain the antiinflammatory effect. Visualization of docked inhibitors in COX-2 enzyme reveals that the carboxylate group of NSAIDs is located in a favorable position to interact with the guanidinium group of Arginine 120 and $\mathrm{OH}$ of Tyrosine 355 (Chakraborti and Thilagavathi, 2003).

First of all the main interactions of Indomethacin ${ }^{\circledR}$ with COX-2 were determined and it was found that Indomethacin ${ }^{\circledR}$ formed 5 hydrogen bonds (affinity value of $-73.25 \mathrm{kcal} / \mathrm{mol}$ ). One hydrogen bond with Tyr355 (-OH group) with a distance of $1.84 \AA$, 3 hydrogen bonds with the guanidinium group of Arg120 and the distances were found to be $1.66 \AA, 2.23 \AA$ and $2.56 \AA$. These are the key amino acids acting as a gate for ligand entrance to the COX-2 active side. 
Additionally, indomethacin formed another hydrogen bond with Serine530 with a distance of 2.06 $\AA$ (Figure 2). The phenyl ring of indol moiety was surrounded by the aromatic residues Phenylalanine518 and Valine349. The other phenyl ring was oriented in the hydrophobic cleft formed by Tryptophan387, Leucine531 and Alanine527.

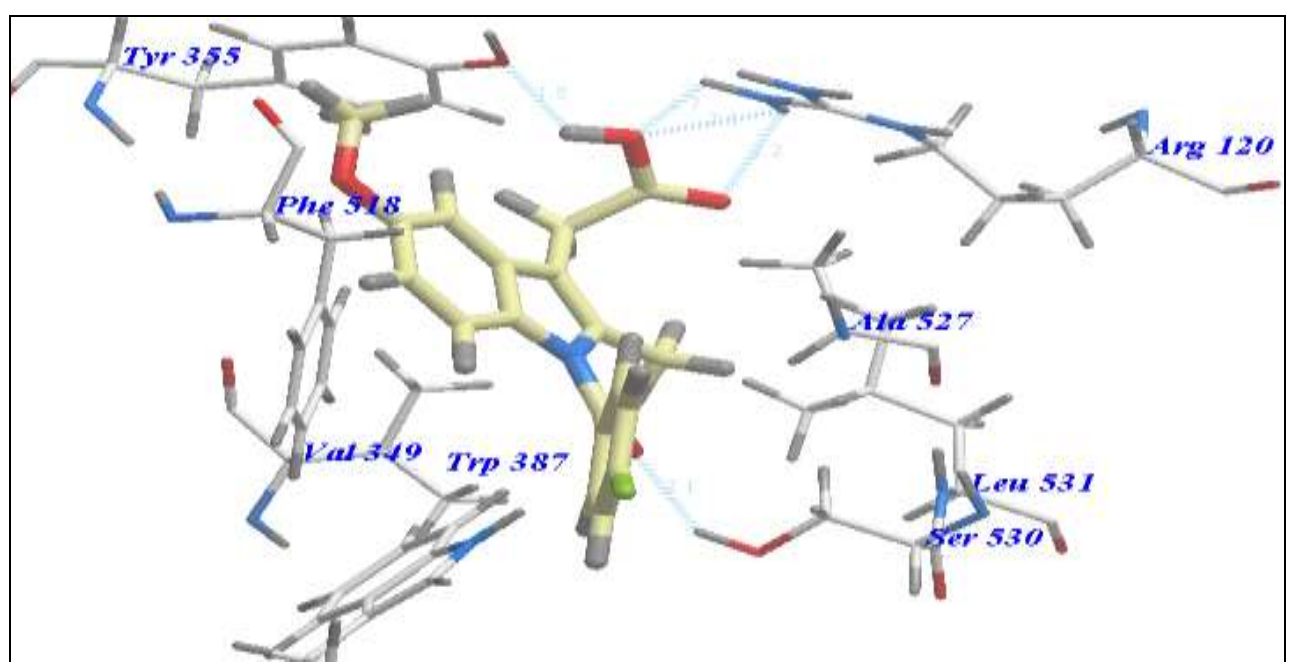

Figure (2): Predicted binding mode for indomethacin with 1PXX. H-bonds are indicated by dotted lines. Hydrogen (gray), nitrogen (blue), oxygen (red) and chloride (green).

The obtained results indicated that all studied ligands have a similar position and orientation inside the putative binding site of COX-2 receptor (PDB code 1PXX). In addition, the affinity of any small molecule can be considered as a unique tool in the field of drug design. As there is a relationship between the affinity of organic molecules and the free energy of binding and this can contribute in prediction and interpretation of the activity of organic compounds toward a specific target protein. The obtained results of the free energy of binding $(\Delta G)$ explained that; most of these compounds had good binding affinity towards the receptor and the computed values reflect the overall trend (Table 1).

The proposed binding mode of compound $8_{\mathrm{a}}$, (affinity value of $-108.69 \mathrm{kcal} / \mathrm{mol}$ ) was virtually the same as that of indomethacin (Figure 3) where tetrahydropyrimidone moiety was stabilized by formation of one hydrogen bond with the guanidinium group of $\operatorname{Arg} 120$ (2.71 $\AA$ ), one hydrogen bond with the residue Pro $86(1.64 \AA)$ and three hydrogen bonding interaction with the guanidinium group of Arg513 (1.52 $\AA, 2.43 \AA$ and $2.57 \AA)$. The 6-iodo-2-phenylquinazolin4(3H)-one moiety was oriented in the hydrophobic cleft formed by Phe518, Val349, Trp387, Leu531 and Ala527 (Figure 3). These interactions of compound $8_{a}$ may explain the highest binding free energy and anti-inflammatory activity

Moreover the proposed binding mode of compounds $12_{\mathrm{b}}$ (affinity value of $-103.53 \mathrm{kcal} / \mathrm{mol}$ ) revealed that 6-iodo-2-phenylquinazolin-4(3H)-one moiety was oriented in the hydrophobic cleft formed by Phe518, Val349, Trp387, Leu531 and Ala527. It formed hydrogen bond with the guanidinium group of Arg 120 (2.5 $\AA$ ). The 2-amino-3-cyano- $4 H$-pyrane moiety was stabilized by formation of four hydrogen bonds. The 2-amino group formed one hydrogen bond with Ala516 (2.66 $\AA$ ) and two hydrogen bonds with Phe518 (1.53 $\AA$ and $2.59 \AA)$. The 3-cyano group formed one hydrogen bond with Ala516 (1.17 $\AA$ ). The intermediate phenyl was oriented in the hydrophobic cleft formed by Phe518 and Tyr355 (Figure 4). 


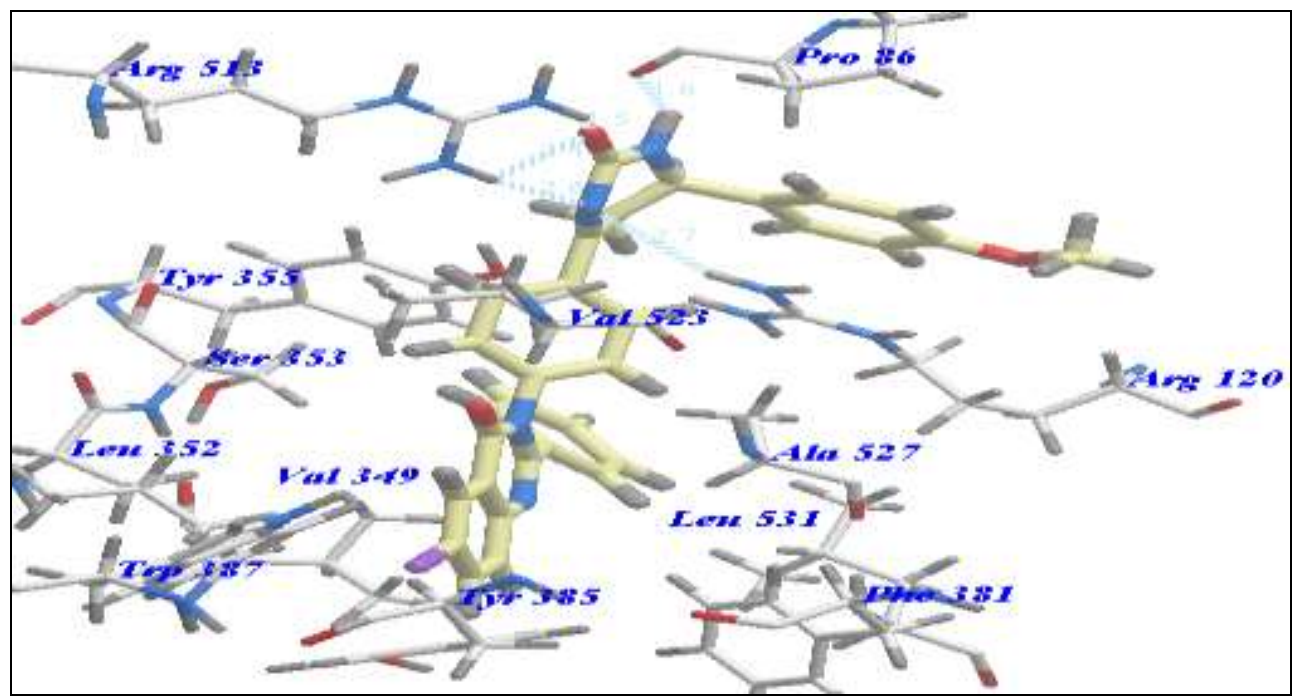

Figure (3): Predicted binding mode for compound $8_{a}$ with 1PXX.

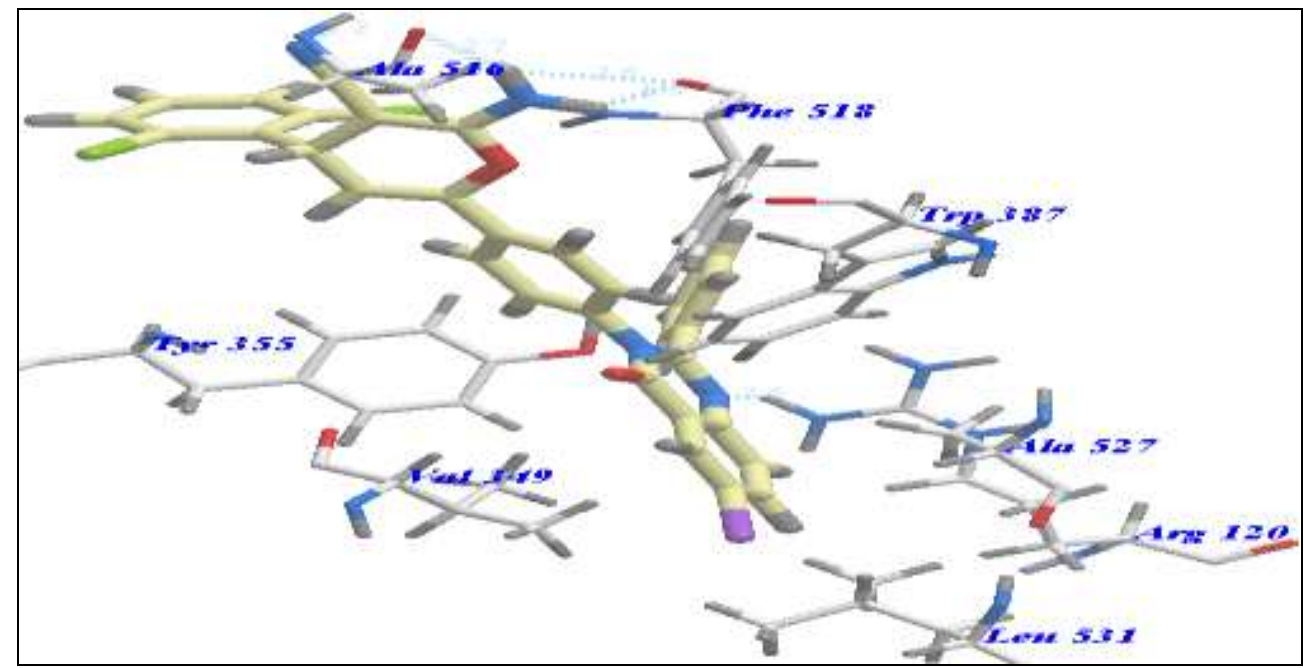

Figure (4): Predicted binding mode for compounds $12_{\mathrm{b}}$ with $1 \mathrm{PXX}$.

Table (1): The calculated $\Delta \mathrm{G}$ (free energy of binding) and binding affinities for the ligands

\begin{tabular}{|c|c|c|c|}
\hline Compound & $\Delta \mathrm{G}\left[\mathrm{kcal} \mathrm{mol}^{-1}\right]$ & Compound & $\left.\Delta \mathrm{G}^{-1} \mathrm{kcal} \mathrm{mol}^{-1}\right]$ \\
\hline $\mathbf{4}$ & -64.90 & $\mathbf{1 0}_{\mathbf{b}}$ & -103.15 \\
\hline $\mathbf{5}_{\mathbf{a}}$ & -86.83 & $\mathbf{1 0}_{\mathbf{c}}$ & -83.14 \\
\hline $\mathbf{5}_{\mathbf{b}}$ & -91.80 & $\mathbf{1 0}_{\mathbf{d}}$ & -84.18 \\
\hline $\mathbf{5}_{\mathbf{c}}$ & -79.99 & $\mathbf{1 0}_{\mathbf{e}}$ & -73.32 \\
\hline $\mathbf{5}_{\mathbf{d}}$ & -85.12 & $\mathbf{1 1}_{\mathbf{a}}$ & -83.44 \\
\hline $\mathbf{5}_{\mathbf{e}}$ & -81.50 & $\mathbf{1 1}_{\mathbf{b}}$ & -84.60 \\
\hline $\mathbf{6}_{\mathbf{a}}$ & -84.60 & $\mathbf{1 1}_{\mathbf{c}}$ & -68.79 \\
\hline $\mathbf{6}_{\mathbf{b}}$ & -82.05 & $\mathbf{1 1}_{\mathbf{d}}$ & -71.75 \\
\hline $\mathbf{7}$ & -97.35 & $\mathbf{1 1}_{\mathbf{e}}$ & -83.71 \\
\hline $\mathbf{8}_{\mathbf{a}}$ & -108.69 & $\mathbf{1 2}_{\mathbf{a}}$ & -82.07 \\
\hline $\mathbf{8}_{\mathbf{b}}$ & -99.31 & $\mathbf{1 2}_{\mathbf{b}}$ & -103.53 \\
\hline $\mathbf{9}_{\mathbf{a}}$ & -86.34 & $\mathbf{1 2}_{\mathbf{c}}$ & -97.72 \\
\hline $\mathbf{9}_{\mathbf{b}}$ & -83.57 & $\mathbf{1 2}_{\mathbf{d}}$ & -85.21 \\
\hline $\mathbf{9}_{\mathbf{c}}$ & -98.12 & indomethacin & -73.25 \\
\hline $\mathbf{1 0}_{\mathbf{a}}$ & -84.43 & & \\
\hline
\end{tabular}




\section{Biological evaluation}

\section{Anti-inflammatory screening}

The anti-inflammatory activity of nineteen of the newly synthesized compounds: $\mathbf{5}_{\mathbf{b}}, \mathbf{6}_{\mathbf{a}}, \mathbf{6}_{\mathbf{b}}$, $\mathbf{7}, \mathbf{8}_{\mathrm{a}}, \mathbf{8}_{\mathrm{b}}, \mathbf{9}_{\mathrm{c}}, \mathbf{1 0}, \mathbf{1 2}_{\mathrm{b}}$ and $\mathbf{1 2} \mathrm{c}$ were evaluated by applying carrageenan-induced paw edema bioassay in rats using indomethacin as a reference standard (Winter $\boldsymbol{e t}$ al., 1962). As shown in (Table 2), administration of tested compounds, 60 minutes prior to carrageenan injection, at a dose of $10 \mathrm{mg} / \mathrm{kg}$ body weight caused significant inhibition of paw edema response. At the end of this step, the two legs of each rat compared to each other by weight (one reference and the other which treated with the studied drug).

Compounds $\mathbf{8}_{\mathbf{a}}, \mathbf{8}_{\mathrm{b}}, \mathbf{9}_{\mathrm{c}}, \mathbf{1 0}_{\mathrm{b}}, \mathbf{1 2}_{\mathrm{b}}$ and $\mathbf{1 2}_{\mathrm{c}}$ caused significant decrease in paw edema after $1 \mathrm{~h}$ after carrageenan administration and continued to the fourth hour, while $\mathbf{7}$ gave its response after 1 $\mathrm{h}$ of administration and continued to the third hour. Compounds $\mathbf{5}_{\mathbf{b}}$ showed its effect after $1 \mathrm{~h}$ of administration and continued to the second hour. On the other hand compounds $\mathbf{6}_{\mathbf{a}}$ and $\mathbf{6}_{\mathbf{b}}$ showed their effect only after $1 \mathrm{~h}$ of administration and were considered to be inactive towards carrageenan-induced edema in comparison to the standard reference indomethacin which markedly and significantly inhibited the paw edema after 1, 2, 3, $4 \mathrm{~h}$ of carrageenan injection (Figure 5).

Compounds $\mathbf{8}_{\mathrm{a}}, \mathbf{8}_{\mathrm{b}}, \mathbf{9}_{\mathrm{c}}, \mathbf{1 0}_{\mathrm{b}}, \mathbf{1 2}_{\mathrm{b}}$ and $\mathbf{1 2}_{\mathrm{c}}$ caused maximum inhibitory effects at all detected time intervals when compared with the standard group. Nevertheless, compounds $\mathbf{6}_{\mathbf{a}}$ and $\mathbf{6}_{\mathbf{b}}$ showed poor effect as anti-inflammatory agents. Other compounds, $\mathbf{5}_{\mathbf{b}}$ and $\mathbf{7}$ displayed moderate to good effects on inhibitory properties.

Structure-activity correlation based on the obtained results indicates that, modifications of quinazolinone nucleus at 3rd position by incorporation of tetrahydropyrimidin-2-ones moiety specially when substituted with 4-methoxyphenyl moiety exhibited the maximum antiinflammatory activity with potencies equal to $115.7,95.4,98.4$ and $95.9 \%$ of that of indomethacin at $1 \mathrm{~h}, 2 \mathrm{~h}, 3 \mathrm{~h}$ and $4 \mathrm{~h}$ after carrageenan injection respectively (Figure 6). Moreover incorporation of pyran moiety substituted with 2,6-dichlorophenyl showed potencies equal to $101.9,81.7,90.6$ and $89.5 \%$. On the other hand incorporation of pyridone moiety substituted with 2,4-dichlorophenyl exhibited potencies equal to 84.1, 85.4, 85.3 and $75.3 \%$ of that of indomethacin at the same time intervals.

Table (2): Anti-inflammatory effect of the selected compounds

\begin{tabular}{|c|c|c|c|c|c|c|c|c|}
\hline \multirow[b]{2}{*}{ Group } & \multicolumn{8}{|c|}{ Mean swelling volume (ml; percentage inhibition of edema) } \\
\hline & $1 \mathrm{~h}$ & $\begin{array}{c}\text { Potency } \\
(\%)\end{array}$ & $2 \mathbf{h}$ & $\begin{array}{c}\text { Potency } \\
(\%)\end{array}$ & $3 \mathbf{h}$ & $\begin{array}{c}\text { Potency } \\
(\%)\end{array}$ & $4 \mathrm{~h}$ & $\begin{array}{c}\text { Potency } \\
(\%)\end{array}$ \\
\hline $\begin{array}{c}\text { Control } \\
\text { ( carrageenan) }\end{array}$ & 0.980 & - & 1.354 & _- & 1.556 & - & 1.411 & - \\
\hline $\mathbf{5}_{\mathbf{b}}$ & $\begin{array}{l}0.678 \\
(30.8)\end{array}$ & 59.8 & $\begin{array}{l}0.950 \\
(29.8)\end{array}$ & 50.5 & $\begin{array}{l}1.200 \\
(22.9)\end{array}$ & 37.1 & $\begin{array}{l}1.241 \\
(12.0)\end{array}$ & 20.9 \\
\hline$\overline{6}$ & $\begin{array}{l}0.712 \\
(27.3)\end{array}$ & 53.0 & $\begin{array}{l}1.118 \\
(17.4)\end{array}$ & 29.5 & $\begin{array}{l}1.239 \\
(20.4)\end{array}$ & 33.0 & $\begin{array}{l}1.298 \\
(8.0)\end{array}$ & 13.9 \\
\hline $\mathbf{6}_{\mathrm{b}}$ & $\begin{array}{l}0.724 \\
(26.1)\end{array}$ & 50.7 & $\begin{array}{l}1.122 \\
(17.1)\end{array}$ & 28.9 & $\begin{array}{l}1.235 \\
(20.6)\end{array}$ & 33.3 & $\begin{array}{l}1.311 \\
(7.1)\end{array}$ & 12.4 \\
\hline 7 & $\begin{array}{l}0.578 \\
(41.0)\end{array}$ & 79.6 & $\begin{array}{l}0.820 \\
(39.4)\end{array}$ & 66.8 & $\begin{array}{l}0.983 \\
(36.8)\end{array}$ & 59.5 & $\begin{array}{l}1.020 \\
(27.7)\end{array}$ & 48.3 \\
\hline 8 & $\begin{array}{l}0.396 \\
(59.6)\end{array}$ & 115.7 & $\begin{array}{l}0.592 \\
(56.3)\end{array}$ & 95.4 & $\begin{array}{l}0.610 \\
(60.8)\end{array}$ & 98.4 & $\begin{array}{l}0.634 \\
(55.1)\end{array}$ & 95.9 \\
\hline
\end{tabular}




\begin{tabular}{|c|c|c|c|c|c|c|c|c|}
\hline $\mathbf{8}_{\mathbf{b}}$ & $\begin{array}{c}0.562 \\
(42.7)\end{array}$ & 82.9 & $\begin{array}{c}0.678 \\
(49.9)\end{array}$ & 84.6 & $\begin{array}{c}0.744 \\
(52.2)\end{array}$ & 84.5 & 0.818 & 73.2 \\
& 0.575 & 80.2 & 0.698 & 82.0 & 0.758 & 83.0 & 0.890 & 64.3 \\
& $(41.3)$ & & $(48.4)$ & & $(51.3)$ & & $(36.9)$ & \\
\hline $\mathbf{9}_{\mathbf{c}}$ & 0.559 & 84.1 & 0.672 & 85.4 & 0.736 & 85.3 & 0.801 & 75.3 \\
& $(43.3)$ & & $(50.4)$ & & $(52.7)$ & & $(43.2)$ & \\
\hline $\mathbf{1 0}_{\mathbf{b}}$ & 0.466 & 101.9 & 0.701 & 81.7 & 0.684 & 90.6 & 0.686 & 89.5 \\
& $(52.5)$ & & $(48.2)$ & & $(56.0)$ & & $(51.4)$ & \\
\hline $\mathbf{1 2}_{\mathbf{b}}$ & 0.611 & 73.0 & 0.732 & 77.8 & 0.779 & 80.7 & 0.921 & 60.5 \\
& $(37.6)$ & & $(45.9)$ & & $(49.9)$ & & $(34.7)$ & \\
\hline $\mathbf{1 2}_{\mathbf{c}}$ & 0.475 & 100 & $\begin{array}{c}0.555 \\
(59.0)\end{array}$ & 100 & $\begin{array}{c}0.594 \\
(61.8)\end{array}$ & 100 & 0.600 & 100 \\
& $(51.5)$ & & $(57.4)$ & \\
\hline
\end{tabular}

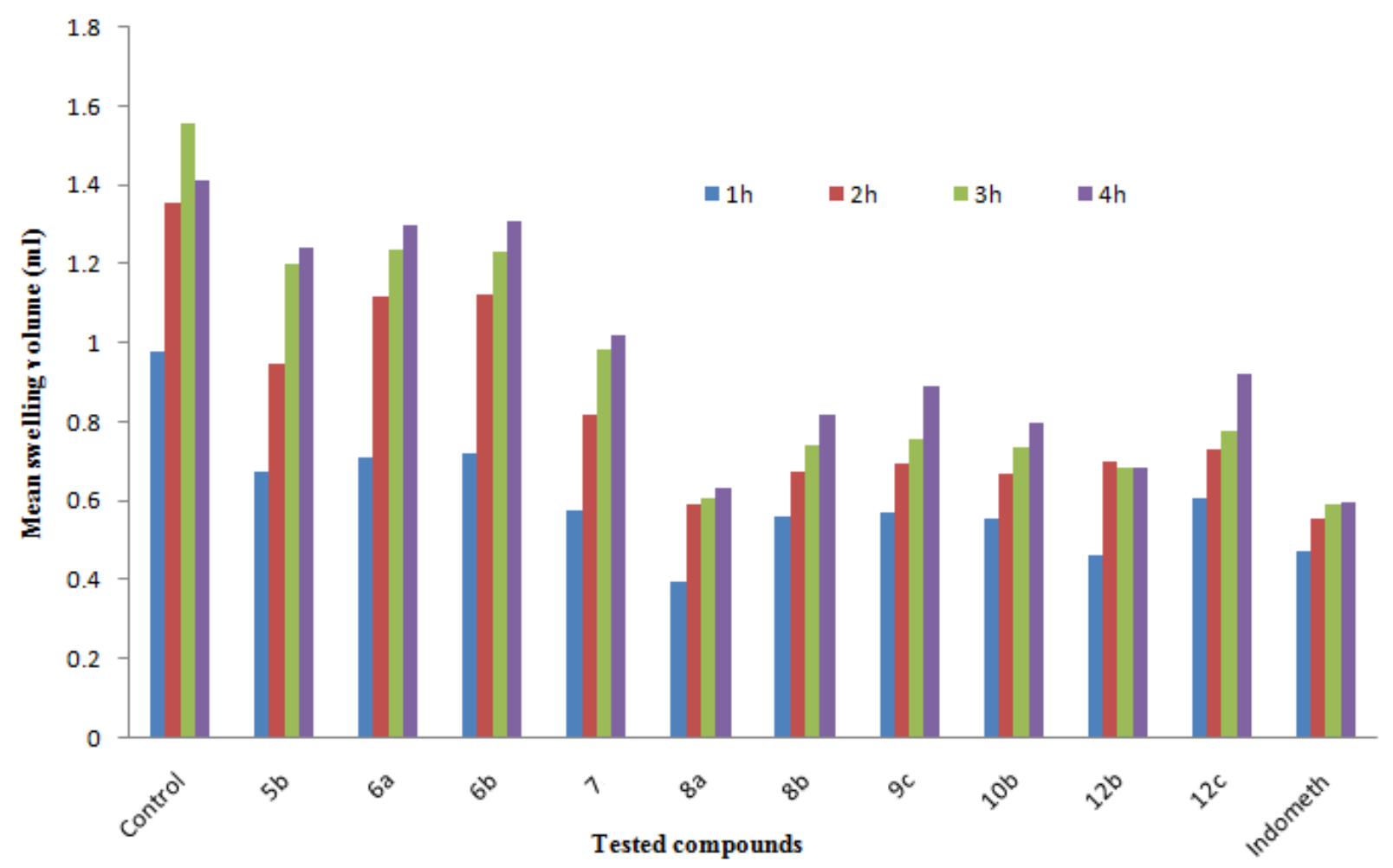

Figure (5): Mean edema volume (ml) of the tested compounds at successive time intervals.

\section{Gastric ulcerogenic studies}

The ulcerogenic effect of the tested derivatives was evaluated (Amr and Abdulla 2006). According to Table 3, it has been found that all compounds have very little ulcerogenic effect with better safety margin in comparison to indomethacin. Compounds $\mathbf{5}_{b}, \mathbf{6}_{b}, \mathbf{8}_{a}, \mathbf{9}_{c}$ and $\mathbf{1 2}_{b}$ have very little ulcerogenic effects with better safety margin. Compounds $\mathbf{6}_{\mathbf{a}}, \mathbf{9}_{\mathrm{c}}, \mathbf{1 0}_{\mathrm{b}}$ and $\mathbf{1 2}_{\mathrm{c}}$ have moderate ulcerogenic effects. On the other hand compound 7 resulted in ulcer lesions in many of the experimental rats. Therefore, the potential medicinal value of these compounds as antiinflammatory, that they have better safety margin than indomethacin on gastric mucosa. 
Table (3): Ulcerogenic effect of the selected compounds

\begin{tabular}{|c|c|c|c|c|c|}
\hline Group & $\begin{array}{c}\text { Number } \\
\text { of } \\
\text { lesions/rat } \\
\text { X- } \pm \text { S.E. }\end{array}$ & $\begin{array}{c}\text { Severity } \\
\text { of } \\
\text { lesions/rat } \\
\text { X- } \pm \text { S.E. }\end{array}$ & Group & $\begin{array}{c}\text { Number } \\
\text { of } \\
\text { lesions/rat } \\
\text { X- } \pm \text { S.E. }\end{array}$ & $\begin{array}{c}\text { Severity } \\
\text { of } \\
\text { lesions/rat } \\
\text { X- } \pm \text { S.E. }\end{array}$ \\
\hline $\begin{array}{c}\text { Control } \\
\text { (ethanol) }\end{array}$ & $34.5 \pm 2.0$ & $81.3 \pm 2.0$ & $\mathbf{8}_{\mathbf{b}}$ & $10.3 \pm 1.8$ & $13.9 \pm 1.8$ \\
\hline $\mathbf{5}_{\mathbf{b}}$ & $4.6 \pm 0.6$ & $7.2 \pm 0.8$ & $\mathbf{9}_{\mathbf{c}}$ & $3.6 \pm 0.2$ & $14.2 \pm 0.9$ \\
\hline $\mathbf{6}_{\mathbf{a}}$ & $7.5 \pm 0.9$ & $9.1 \pm 0.5$ & $\mathbf{1 0}_{\mathbf{b}}$ & $7.5 \pm 1.4$ & $6.7 \pm 0.8$ \\
\hline $\mathbf{6}_{\mathbf{b}}$ & $5.8 \pm 0.8$ & $8.4 \pm 0.5$ & $\mathbf{1 2}_{\mathbf{b}}$ & $3.2 \pm 0.4$ & $8.8 \pm 0.9$ \\
\hline $\mathbf{7}$ & $12.8 \pm 1.8$ & $15.3 \pm 1.8$ & $\mathbf{1 2}_{\mathbf{c}}$ & $9.3 \pm 1.2$ & $9.5 \pm 0.8$ \\
\hline $\mathbf{8}_{\mathbf{a}}$ & $4.3 \pm 0.7$ & $6.3 \pm 0.8$ & indomethacin & $16.8 \pm 1.3$ & $21.3 \pm 1.8$ \\
\hline
\end{tabular}

Statistical comparison of the difference between the control and treated groups is indicated by asterisks, $\mathrm{p}<0.001$ (Student's $t$-test)

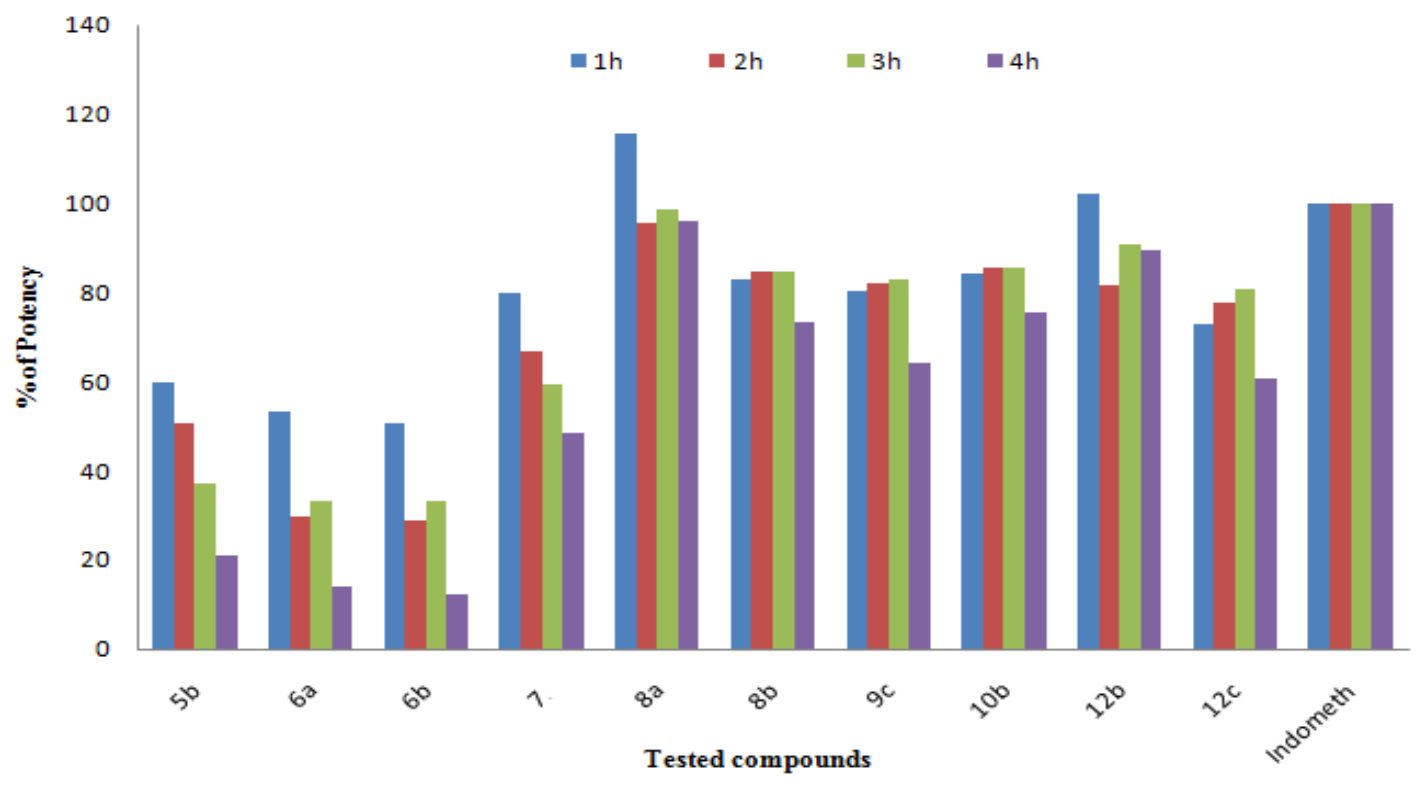

Figure (6): Anti-inflammatory potencies of the tested compounds relative to indomethacin.

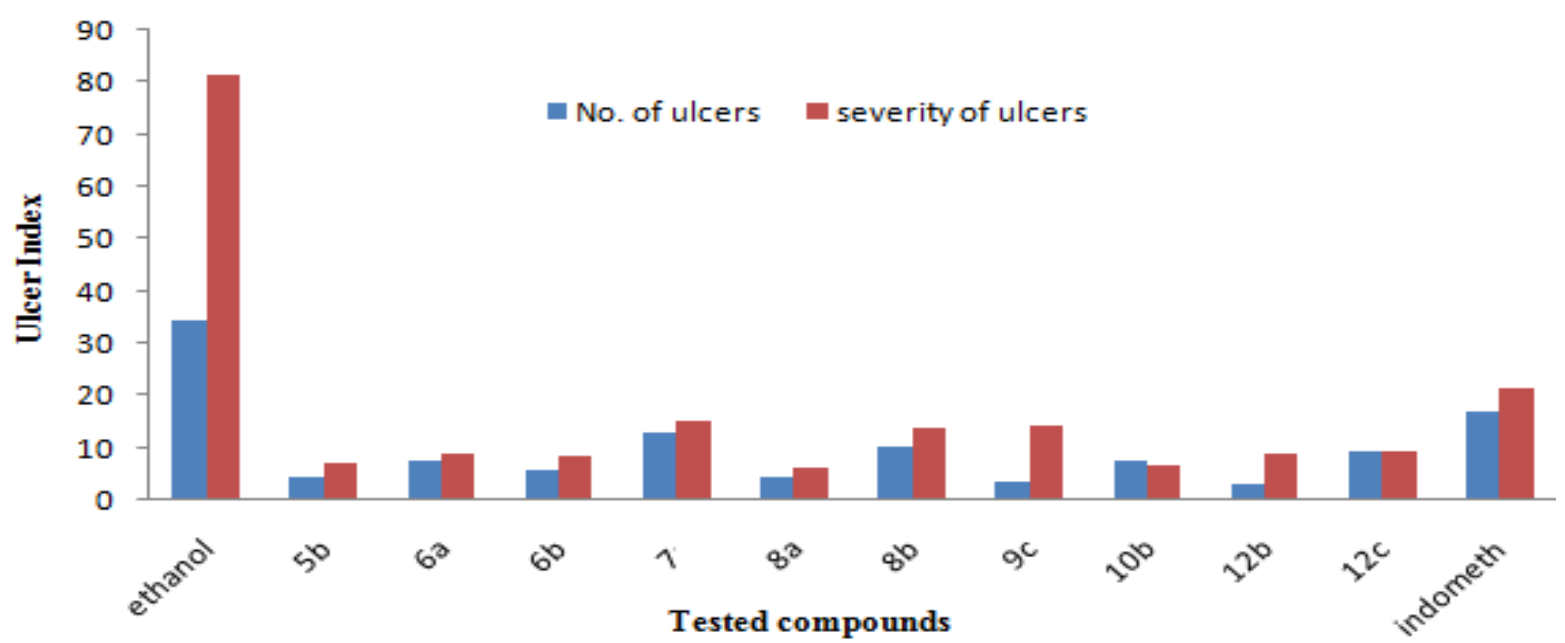

Figure (7): Ulcerogenic effects of the tested compounds relative to indomethacin. 


\section{MATERIALS AND METHODS}

\section{Chemistry}

All melting points were carried out by open capillary method on a Gallen kamp Melting point apparatus at faculty of pharmacy Al-Azhar University and were uncorrected. The infrared spectra were recorded on pye Unicam SP 1000 IR spectrophotometer at Pharmaceutical Analytical Unit, Faculty of Pharmacy, Al-Azhar University using potassium bromide disc technique. Proton magnetic resonance ${ }^{1}$ HNMR spectra were recorded on a jeol 400 MHZ-NMR spectrometer at Microanalytical Center, Cairo University and Microanalytical Center, Asuit University. TMS was used as internal standard and chemical shifts were measured in $\delta$ scale (ppm). The mass spectra were recorded on Varian MAT 311-A (70 e.v.) at Regional Center for Mycology and Biotechnology, Al-Azhar University and Direct Inlet unit (DI-50) of SHIMADZU GC/MSQP5050A at Microanalytical Center, Cairo University. Elemental analyses (C, H, N) were performed on a CHN analyzer at Regional Center for Mycology and Biotechnology, Al-Azhar University. All compounds were within \pm 0.4 of the theoretical values. The reactions were monitored by thin-layer chromatography (TLC) using TLC sheets precoated with UV fluorescent silica gel Merck 60 F254 plates and were visualized using UV lamp and different solvents as mobile phases.

5-iodoanthranilic acid (1), $\mathrm{N}$-benzoyl-5-iodoanthranilic acid (2) and 6-iodo-2-phenyl-4H-3,1benzoxazin-4-one (3) were obtained according to the reported procedures.

\subsubsection{3-(4-Acetylphenyl)-6-iodo-2-phenylquinazolin-4(3H)-one (4)}

A mixture of compound (3) $(3.49 \mathrm{~g}, 0.01 \mathrm{~mol})$ and 4 -aminoacetophenone $(1.35 \mathrm{~g}, 0.01 \mathrm{~mol})$ in acetic acid glacial $(20 \mathrm{ml})$ was refluxed for $4 \mathrm{~h}$. After cooling, the reaction mixture was poured carefully portion wise onto ice-water $(300 \mathrm{ml})$ while stirring and the separated solid was filtered, dried and crystallized from ethanol twice to give the corresponding acetyl derivative (4).

Yield, $82 \%$; m.p.: $230-2^{\circ} \mathrm{C}$. Analysis for $\mathrm{C}_{22} \mathrm{H}_{15} \mathrm{IN}_{2} \mathrm{O}_{2}$ (m. w. 466); Calcd.: C, 56.67; H, 3.24; N, 6.01. Found: $\mathrm{C}, 56.68 ; \mathrm{H}, 3.27 ; \mathrm{N}, 6.12$. IR $\left(\mathrm{KBr}, \mathrm{cm}^{-1}\right): 3075$ ( $\mathrm{CH}$ aromatic), 2970 (CH aliphatic), $1760\left(\mathrm{C}=\mathrm{O}\right.$ of acetyl), $1690\left(\mathrm{C}=\mathrm{O}\right.$ of quinazolinone). ${ }^{1} \mathrm{HNMR}\left(\mathrm{DMSO}-\mathrm{d}_{6}, \mathrm{ppm}\right): 2.5\left(\mathrm{~s}, 3 \mathrm{H}, \mathrm{CH}_{3}\right)$, 7.49-8.09 (m, $12 \mathrm{H}$, aromatic protons). MS (m/z): $467\left(\mathrm{M}^{+1}, 4.58 \%\right), 466\left(\mathrm{M}^{+}, 16.36 \%\right), 465$ (11.39\%), 350 (13.66\%), 105 (35.70\%), 77(100\%).

\subsubsection{6-Iodo-2-phenyl-3-(4-(3-(substituted)acryloyl)phenyl)quinazolin-4(3H)-one (chalcones) $\left(5_{a-e}\right)$}

General method:

To a mixture of the ketone $(4)(0.93 \mathrm{~g}, 0.002 \mathrm{~mol})$ and the appropriate aromatic aldehyde $(0.002 \mathrm{~mol})$ in ethyl alcohol $(10 \mathrm{ml}), 5 \% \mathrm{NaOH}$ in ethyl alcohol $(10 \mathrm{ml})$ was added drop wise within 15 minutes. The reaction mixture was refluxed for 3 hours, then cooled and the formed precipitate was filtered, air dried and then recrystallized from ethanol to give the corresponding chalcones $\left(\mathbf{5}_{\mathrm{a}-\mathrm{e}}\right)$ respectively.

\subsubsection{6-Iodo-2-phenyl-3-(4-(3-(4-methoxyphenyl)acryloyl)phenyl)quinazolin-4(3H)-one $\left(5_{a}\right)$} Yield, $89 \%$; m.p.: $165-7^{\circ} \mathrm{C}$. Analysis for $\mathrm{C}_{30} \mathrm{H}_{21} \mathrm{IN}_{2} \mathrm{O}_{3}$ (m. w. 584); Calcd.: C, 61.66; H, 3.62; N, 4.79. Found: C, 62.14; H, 4.08; N, 4.69. IR ( $\left.\mathrm{KBr}, \mathrm{cm}^{-1}\right): 3067(\mathrm{CH}$ aromatic), $1722(\mathrm{C}=\mathrm{O}$ of $\alpha, \beta-$ unsaturated ketone), $1664\left(\mathrm{C}=\mathrm{O}\right.$ of quinazolinone). ${ }^{1} \mathrm{HNMR}$ (DMSO-d $\left.\mathrm{d}_{6}, \mathrm{ppm}\right): 4.33$ (s, 3H, $\left.\mathrm{OCH}_{3}\right), 7.53-8.52(\mathrm{~m}, 18 \mathrm{H}, \mathrm{CH}=\mathrm{CH}$ overlapped with the aromatic protons).

\subsubsection{3-(4-(3-(2,4-Dichlorophenyl)acryloyl)phenyl)-6-iodo-2-phenylquinazolin-4(3H)-one} $\left(5_{b}\right)$ 
Yield, $83 \%$; m.p.: $184-6^{\circ} \mathrm{C}$. Analysis for $\mathrm{C}_{29} \mathrm{H}_{17} \mathrm{Cl}_{2} \mathrm{IN}_{2} \mathrm{O}_{2}$ (m. w. 623); Calcd.: C, 55.88; H, 2.75; $\mathrm{N}$, 4.49. Found: C, 55.86; H, 2.76; N, 4.60. IR ( $\left.\mathrm{KBr}, \mathrm{cm}^{-1}\right): 3071(\mathrm{CH}$ aromatic $), 1725(\mathrm{C}=\mathrm{O}$ of $\alpha, \beta$-unsaturated ketone), $1655\left(\mathrm{C}=\mathrm{O}\right.$ of quinazolinone). $\mathrm{MS}(\mathrm{m} / \mathrm{z}): 622\left(\mathrm{M}^{-1}, 1.32 \%\right), 350$ (63.10\%), 306 (21.44\%), 77 (100\%), 50 (72.17\%).

\subsubsection{3-(4-(3-(2,6-Dichlorophenyl)acryloyl)phenyl)-6-iodo-2-phenylquinazolin-4(3H)-one $\left(5_{c}\right)$}

Yield, $87 \%$; m.p.: $182-4^{\circ} \mathrm{C}$. Analysis for $\mathrm{C}_{29} \mathrm{H}_{17} \mathrm{Cl}_{2} \mathrm{IN}_{2} \mathrm{O}_{2}$ (m. w. 623); Calcd.: C, 55.88; H, 2.75; $\mathrm{N}, 4.49$. Found: $\mathrm{C}, 55.91 ; \mathrm{H}, 2.78 ; \mathrm{N}, 4.58$. IR $\left(\mathrm{KBr}, \mathrm{cm}^{-1}\right): 3072(\mathrm{CH}$ aromatic $), 1728(\mathrm{C}=\mathrm{O}$ of $\alpha, \beta$-unsaturated ketone), $1655\left(\mathrm{C}=\mathrm{O}\right.$ of quinazolinone). $\mathrm{MS}(\mathrm{m} / \mathrm{z}): 622\left(\mathrm{M}^{-1}, 0.15 \%\right), 352(0.30 \%)$, $306(0.30 \%), 77$ (80.72\%), 50 (100\%).

\subsubsection{6-Iodo-2-phenyl-3-(4-(3-(3,4,5-trimethoxyphenyl)acryloyl)phenyl)quinazolin-4(3H)- one $\left(5_{d}\right)$}

Yield, $80 \%$; m.p.: $192-4^{\circ} \mathrm{C}$. Analysis for $\mathrm{C}_{32} \mathrm{H}_{25} \mathrm{IN}_{2} \mathrm{O}_{5}$ (m. w. 644); Calcd.: C, 59.64; H, 3.91; N, 4.35. Found: C, 59.69; H, 3.90; N, 4.54. IR (KBr, cm $\left.{ }^{-1}\right): 3118(\mathrm{CH}$ aromatic), $1732(\mathrm{C}=\mathrm{O} \alpha, \beta-$ unsaturated ketone), $1681\left(\mathrm{C}=\mathrm{O}\right.$ of quinazolinone). ${ }^{1} \mathrm{HNMR}\left(\mathrm{DMSO}-\mathrm{d}_{6}, \mathrm{ppm}\right): 3.31$ (s, 9H, $\left.3 \mathrm{OCH}_{3}\right), 7.52-8.19\left(\mathrm{~m}, 16 \mathrm{H}, \mathrm{CH}=\mathrm{CH}\right.$ overlapped with the aromatic protons). $\mathrm{MS}(\mathrm{m} / \mathrm{z}): 644\left(\mathrm{M}^{+}\right.$, $3.40 \%), 350$ (42.40\%), 120 (32.72\%), 105 (76.29\%), 77 (100\%).

\subsubsection{6-Iodo-2-phenyl-3-(4-(3-(thiophen-2-yl)acryloyl)phenyl)quinazolin-4(3H)-one $\left(5_{e}\right)$}

Yield, $93 \%$; m.p.: $182-4^{\circ} \mathrm{C}$. Analysis for $\mathrm{C}_{27} \mathrm{H}_{17} \mathrm{IN}_{2} \mathrm{O}_{2} \mathrm{~S}$ (m. w. 560); Calcd.: C, 57.87; H, 3.06; N, 5.00. Found: C, 57.89; H, 3.09; N, 5.18. IR $\left(\mathrm{KBr}, \mathrm{cm}^{-1}\right): 3057(\mathrm{CH}$ aromatic $), 1728(\mathrm{C}=\mathrm{O} \alpha, \beta-$ unsaturated ketone), $1646\left(\mathrm{C}=\mathrm{O}\right.$ of quinazolinone). $\mathrm{MS}(\mathrm{m} / \mathrm{z}): 562\left(\mathrm{M}^{+2}, 1.59 \%\right), 350(17.60 \%)$, $120(2.14 \%), 105(40.69 \%), 77(100 \%)$.

\subsubsection{6-Iodo-2-phenyl-3-(4-(5-substituted-4,5-dihydro-1H-pyrazol-3-yl)phenyl)quinazolin- $4(3 \mathrm{H})$-one $\left(6_{a-b}\right)$}

\section{General method:}

A mixture of the appropriate chalcone (5) $(0.005 \mathrm{~mol})$ and hydrazine hydrate $(2.5 \mathrm{ml}, 0.005$ mol, 98\%) in absolute ethanol (25 ml) was refluxed for 10 hours. After cooling, the separated precipitate was filtered, air dried and recrystallized from ethanol to afford the corresponding pyrazoline derivatives $\left(\mathbf{6}_{\mathbf{a}-\mathbf{b}}\right)$ respectively.

\subsubsection{6-Iodo-3-(4-(5-(4-methoxyphenyl)-4,5-dihydro-1H-pyrazol-3-yl)phenyl)-2-phenyl- quinazolin-4(3H)-one $\left(6_{a}\right)$}

Yield, $76 \%$; m.p.: $235-7^{\circ} \mathrm{C}$. Analysis for $\mathrm{C}_{30} \mathrm{H}_{23} \mathrm{IN}_{4} \mathrm{O}_{2}$ (m. w. 598); Calcd.: C, 60.21; H, 3.87; N, 9.36. Found: C, 60.27; H, 3.84; N, 9.36. IR $\left(\mathrm{KBr}, \mathrm{cm}^{-1}\right): 3255(\mathrm{NH})$, disappearance of absorption band of $\left(\mathrm{C}=\mathrm{O}\right.$ of chalcone), $1665\left(\mathrm{C}=\mathrm{O}\right.$ of quinazolinone). ${ }^{1} \mathrm{HNMR}$ (DMSO-d $\left.\mathrm{d}_{6}, \mathrm{ppm}\right): 1.06$ (dd, $2 \mathrm{H}, \mathrm{CH}_{2}$ of pyrazoline), $3.35\left(\mathrm{~s}, 3 \mathrm{H}, \mathrm{OCH}_{3}\right), 3.45(\mathrm{t}, 1 \mathrm{H}, \mathrm{CH}$ of pyrazoline $), 7.26-8.51(\mathrm{~m}, 16 \mathrm{H}$, aromatic protons), $15.49(\mathrm{~s}, 1 \mathrm{H}, \mathrm{NH})$. MS (m/z): $597\left(\mathrm{M}^{-1}, 0.17 \%\right), 350(3.82 \%), 105$ (26.81\%), $76(100 \%), 68(1.04 \%), 50(58.28 \%)$.

\subsubsection{6-Iodo-2-phenyl-3-(4-(5-(3,4,5-trimethoxyphenyl)-4,5-dihydro-1H-pyrazol-3-yl)- phenyl)quinazolin-4(3H)-one $\left(6_{b}\right)$}

Yield, 77 \%; m.p.: 261-3 ${ }^{\circ}$ C. Analysis for $\mathrm{C}_{32} \mathrm{H}_{27} \mathrm{IN}_{4} \mathrm{O}_{4}$ (m. w. 658); Calcd.: C, 58.37; H, 4.13; N, 8.51. Found: C, 57.99; H, 4.20; N, 8.91. IR ( $\left.\mathrm{KBr}, \mathrm{cm}^{-1}\right): 3107(\mathrm{NH}), 3050$ (CH aromatic), disappearance of absorption band of $(\mathrm{C}=\mathrm{O}$ of chalcone $), 1660(\mathrm{C}=\mathrm{O}$ of quinazolinone $) .{ }^{1} \mathrm{HNMR}$ (DMSO- $\left.\mathrm{d}_{6}, \mathrm{ppm}\right): 1.04\left(\mathrm{dd}, 2 \mathrm{H}, \mathrm{CH}_{2}\right.$ of pyrazoline), $3.43\left(\mathrm{~s}, 9 \mathrm{H}, 3 \mathrm{OCH}_{3}\right), 3.80(\mathrm{t}, 1 \mathrm{H}, \mathrm{CH}$ of pyrazoline), 7.27-8.51 (m, 14H, aromatic protons), $15.50(\mathrm{~s}, 1 \mathrm{H}, \mathrm{NH})$. 


\subsubsection{3-(4-(1-Acetyl-5-(thiophen-2-yl)-4,5-dihydro-1H-pyrazol-3-yl)phenyl)-6-iodo-2-phenyl- quinazolin-4(3H)-one (7)}

\section{General method:}

A mixture of the appropriate chalcone $\left(\mathbf{5}_{\mathbf{e}}\right)(0.005 \mathrm{~mol})$ and hydrazine hydrate $(2.5 \mathrm{ml}, 0.005$ mol, 98\%) in the presence of acetic acid glacial $(10 \mathrm{ml})$ was refluxed for 6 hours. After cooling, the separated precipitate was filtered, air dried and recrystallized from ethanol to afford the corresponding $N$-acetylpyrazoline derivative (7).

Yield, $82 \%$; m.p.: $151-3^{\circ} \mathrm{C}$. Analysis for $\mathrm{C}_{29} \mathrm{H}_{21} \mathrm{IN}_{4} \mathrm{O}_{2} \mathrm{~S}$ (m. w. 616); Calcd.: C, 56.50; H, 3.43; N, 9.09. Found: C, 56.56; H, 3.48; N, 9.13. IR $\left(\mathrm{KBr}, \mathrm{cm}^{-1}\right): 3044(\mathrm{CH}$ aromatic), $1695(\mathrm{C}=\mathrm{O}$ of acetyl), 1649 (C=O of quinazolinone). ${ }^{1} \mathrm{HNMR}\left(\mathrm{DMSO}-\mathrm{d}_{6}, \mathrm{ppm}\right): 1.02\left(\mathrm{dd}, 2 \mathrm{H}, \mathrm{CH}_{2}\right.$ of pyrazoline $), 1.61(\mathrm{t}, \mathrm{H}, \mathrm{CH}$ of pyrazoline $), 2.60\left(\mathrm{~s}, 3 \mathrm{H}, \mathrm{CH}_{3}\right), 7.76-8.47(\mathrm{~m}, 15 \mathrm{H}$, aromatic protons). MS (m/z): $617\left(\mathrm{M}^{+1}, 1.74 \%\right), 350$ (12.97\%), 105 (84.69\%), 77 (100\%), 63 (24.47\%), 50 $(94.85 \%)$.

\subsubsection{6-Iodo-3-(4-(2-oxo-6-substituted-1,2,5,6-tetrahydropyrimidin-4-yl)phenyl)-2-phenyl- quinazolin-4(3H)-one $\left(8_{a-b}\right)$}

\section{General method:}

A mixture of the appropriate chalcone (5) $(0.005 \mathrm{~mol})$ and urea $(0.5 \mathrm{~g}, 0.005 \mathrm{~mol})$ in ethanol $(20 \mathrm{ml})$ and conc. $\mathrm{HCl}(5 \mathrm{ml})$ was refluxed for 7 hours. The reaction mixture was concentrated to half of its volume, cooled and neutralized with $\mathrm{NH}_{4} \mathrm{OH}$ solution. The precipitated solid was filtered, washed with water, air dried and recrystallized from ethanol to give the corresponding compounds $\left(\boldsymbol{8}_{a-b}\right)$ respectively.

\subsubsection{6-Iodo-3-(4-(6-(4-methoxyphenyl)-2-oxo-1,2,5,6-tetrahydropyrimidin-4-yl)phenyl)-2- phenylquinazolin-4(3H)-one $\left(8_{a}\right)$}

Yield, $76 \%$; m.p.: $153-5^{\circ} \mathrm{C}$. Analysis for $\mathrm{C}_{31} \mathrm{H}_{23} \mathrm{IN}_{4} \mathrm{O}_{3}$ (m. w. 626); Calcd.: C, 59.44; H, 3.70; N, 8.94. Found: C, 59.66; H, 3.37; N, 9.03. IR $\left(\mathrm{KBr}, \mathrm{cm}^{-1}\right): 3112(\mathrm{NH}), 3034(\mathrm{CH}$ aromatic), 1691 (C=O of quinazolinone), 1648 ( $\mathrm{C}=\mathrm{O}$ amidic). ${ }^{1} \mathrm{HNMR}$ (DMSO- $\left.\mathrm{d}_{6}, \mathrm{ppm}\right): 0.85\left(\mathrm{dd}, 2 \mathrm{H}, \mathrm{CH}_{2}\right.$ of pyrimidinone), $1.88\left(\mathrm{t}, 1 \mathrm{H}, \mathrm{CH}\right.$ of pyrimidinone), $3.29\left(\mathrm{~s}, 3 \mathrm{H}, \mathrm{OCH}_{3}\right), 7.07-8.48(\mathrm{~m}, 16 \mathrm{H}$, aromatic protons), $10.75(\mathrm{~s}, 1 \mathrm{H}, \mathrm{NH}) . \mathrm{MS}(\mathrm{m} / \mathrm{z}): 625\left(\mathrm{M}^{-1}, 0.24 \%\right), 350(0.26 \%), 105(16.21 \%)$, $76(32.25 \%), 68(46.42 \%), 56(100 \%)$.

\subsubsection{6-Iodo-3-(4-(2-oxo-6-(thiophen-2-yl)-1,2,5,6-tetrahydropyrimidin-4-yl)phenyl)-2- phenylquinazolin-4(3H)-one $\left(8_{b}\right)$}

Yield, $83 \%$; m.p.: $133-5^{\circ} \mathrm{C}$. Analysis for $\mathrm{C}_{28} \mathrm{H}_{19} \mathrm{IN}_{4} \mathrm{O}_{2} \mathrm{~S}$ (m. w. 602); Calcd.: C, 55.82; H, 3.18; N, 9.30. Found: C, 55.98; H, 2.92; N, 9.42. IR $\left(\mathrm{KBr}, \mathrm{cm}^{-1}\right): 3108(\mathrm{NH}), 3025(\mathrm{CH}$ aromatic), 1695 $\left(\mathrm{C}=\mathrm{O}\right.$ of quinazolinone), $1653(\mathrm{C}=\mathrm{O}$ amidic $) . \mathrm{MS}(\mathrm{m} / \mathrm{z}): 602\left(\mathrm{M}^{+}, 0.03 \%\right), 350(0.06 \%), 105$ (10.71\%), 77 (31.50\%), 68 (19.65\%), 50 (100\%).

\subsubsection{6-Iodo-2-phenyl-3-(4-(6-substituted-2-thioxo-1,2,5,6-tetrahydropyrimidin-4-yl)phenyl)- quinazolin-4(3H)-one $\left(9_{a-c}\right)$}

\section{General method:}

A mixture of the appropriate chalcone (5) $(0.005 \mathrm{~mol})$ and thiourea $(0.38 \mathrm{~g}, 0.005 \mathrm{~mol})$, in the presence of $(0.5 \mathrm{~g})$ of $\mathrm{NaOH}$ was refluxed in ethanol $(25 \mathrm{ml})$ for 6 hours, then concentrated under vacuum and neutralized with diluted $\mathrm{HCl}$. The precipitated material was filtered, washed with water, dried and recrystallized from ethanol to give the corresponding compounds $\left(\boldsymbol{9}_{a-c}\right)$ respectively.

\subsubsection{6-Iodo-3-(4-(6-(4-methoxyphenyl)-2-thioxo-1,2,5,6-tetrahydropyrimidin-4-yl)phenyl)-2- phenylquinazolin-4(3H)-one $\left(9_{a}\right)$}

Yield, 79 \%; m.p.: 231- $3^{\circ} \mathrm{C}$. Analysis for $\mathrm{C}_{31} \mathrm{H}_{23} \mathrm{IN}_{4} \mathrm{O}_{2} \mathrm{~S}$ (m. w. 642); Calcd.: C, 57.95; H, 3.61; N, 
8.72. Found: C, 58.18; H, 3.38; N, 8.86. IR (KBr, $\left.\mathrm{cm}^{-1}\right): 3278(\mathrm{NH}), 3059(\mathrm{CH}$ aromatic), 1656 $\left(\mathrm{C}=\mathrm{O}\right.$ of quinazolinone), $1590(\mathrm{C}=\mathrm{N}), 1250(\mathrm{C}=\mathrm{S}) . \mathrm{MS}(\mathrm{m} / \mathrm{z}): 640\left(\mathrm{M}^{-2}, 4.01 \%\right), 351(7.71 \%), 105$ (39.07\%), 77 (100\%), $63(32.54 \%)$.

\subsubsection{6-Iodo-2-phenyl-3-(4-(2-thioxo-6-(3,4,5-trimethoxyphenyl)-1,2,5,6-tetrahydro- pyrimidin-4-yl)phenyl)quinazolin-4(3H)-one $\left(9_{b}\right)$}

Yield, $83 \%$; m.p.: 257-9 $9^{\circ}$. Analysis for $\mathrm{C}_{33} \mathrm{H}_{27} \mathrm{IN}_{4} \mathrm{O}_{4} \mathrm{~S}$ (m. w. 702); Calcd.: C, 56.42; H, 3.87; N, 7.97. Found: C, 56.62; H, 3.65; N, 8.06. IR $\left(\mathrm{KBr}, \mathrm{cm}^{-1}\right): 3219(\mathrm{NH}), 3073$ (CH aromatic), 1657 $\left(\mathrm{C}=\mathrm{O}\right.$ of quinazolinone), $1591(\mathrm{C}=\mathrm{N}), 1244(\mathrm{C}=\mathrm{S}) .{ }^{1} \mathrm{HNMR}\left(\mathrm{DMSO}-\mathrm{d}_{6}, \mathrm{ppm}\right): 1.02\left(\mathrm{dd}, 2 \mathrm{H}, \mathrm{CH}_{2}\right.$ of pyrimidinthione), $1.61(\mathrm{t}, \mathrm{H}, \mathrm{CH}$ of pyrimidinthione $), 3.78\left(\mathrm{~s}, 9 \mathrm{H}, 3 \mathrm{OCH}_{3}\right), 6.76-8.29(\mathrm{~m}, 14 \mathrm{H}$, aromatic protons), $12.07(\mathrm{~s}, 1 \mathrm{H}, \mathrm{NH})$.

\subsubsection{6-Iodo-2-phenyl-3-(4-(6-(thiophen-2-yl)-2-thioxo-1,2,5,6-tetrahydropyrimidin-4- yl)phenyl)quinazolin-4(3H)-one $\left(9_{c}\right)$}

Yield, $82 \%$; m.p.: 213-5 $5^{\circ}$. Analysis for $\mathrm{C}_{28} \mathrm{H}_{19} \mathrm{IN}_{4} \mathrm{OS}_{2}$ (m. w. 618); Calcd.: C, 54.37; H, 3.10; N, 9.06. Found: C, 54.56; H, 2.76; N, 9.05. IR $\left(\mathrm{KBr}, \mathrm{cm}^{-1}\right): 3275(\mathrm{NH}), 3073(\mathrm{CH}$ aromatic), 1655 $\left(\mathrm{C}=\mathrm{O}\right.$ of quinazolinone), $1549(\mathrm{C}=\mathrm{N}), 1244(\mathrm{C}=\mathrm{S}) . \mathrm{MS}(\mathrm{m} / \mathrm{z}): 616\left(\mathrm{M}^{-2}, 0.01 \%\right), 350(0.09 \%), 105$ (52.04\%), 76 (98.18\%), $62(15.72 \%), 50(100 \%)$.

\subsubsection{6-(4-(6-Iodo-4-oxo-2-phenylquinazolin-3(4H)-yl)phenyl)-2-oxo-4-substituted-1,2- dihydropyridine-3-carbonitrile (10 $\left.10_{a-e}\right)$}

\section{General method:}

A mixture of ketone (4) $(0.93 \mathrm{~g}, 0.002 \mathrm{~mol})$, ethyl cyanoacetate $(0.23 \mathrm{ml}, 0.002 \mathrm{~mol})$, anhydrous ammonium acetate $(1.24 \mathrm{~g}, 0.016 \mathrm{~mol})$ and the appropriate aldehyde $(0.002 \mathrm{~mol})$ in 10 $\mathrm{ml}$ of n-butanol was refluxed for 6 hours. The reaction mixture was concentrated to half of its volume under reduced pressure. After cooling, the formed precipitate was filtered, air dried and crystallized from ethanol to afford the corresponding compounds $\left(\mathbf{1 0}_{\mathrm{a}-\mathrm{e}}\right)$ respectively.

\subsubsection{6-(4-(6-Iodo-4-oxo-2-phenylquinazolin-3(4H)-yl)phenyl)-4-(4-methoxyphenyl)-2-oxo- 1,2-dihydropyridine-3-carbonitrile $\left(10_{a}\right)$}

Yield, $80 \%$; m.p.: 337-9 ${ }^{\circ} \mathrm{C}$. Analysis for $\mathrm{C}_{33} \mathrm{H}_{21} \mathrm{IN}_{4} \mathrm{O}_{3}$ (m. w. 648); Calcd.: C, 61.12; H, 3.26; N, 8.64. Found: C, 61.16; H, 3.28; N, 8.77. IR ( KBr, cm $\left.\mathrm{cm}^{-1}\right): 3339$ (OH enolic of pyridone), $3058(\mathrm{CH}$ aromatic), $2219(\mathrm{CN}), 1674\left(\mathrm{C}=\mathrm{O}\right.$ quinazolinone). ${ }^{1} \mathrm{HNMR}$ (DMSO-d $\left.\mathrm{d}_{6}, \delta, \mathrm{ppm}\right): 3.29(\mathrm{~s}, 3 \mathrm{H}$, $\left.\mathrm{OCH}_{3}\right), 7.56-8.19(\mathrm{~m}, 17 \mathrm{H}$, aromatic protons including $1 \mathrm{H}$ of pyridone $), 10.83(\mathrm{~s}, 1 \mathrm{H}, \mathrm{NH})\left(\mathrm{D}_{2} \mathrm{O}\right.$ exchangeable), $11.31\left(\mathrm{~s}, 1 \mathrm{H}, \mathrm{OH}\right.$ of resonance) $\left(\mathrm{D}_{2} \mathrm{O}\right.$ exchangeable $) . \mathrm{MS}(\mathrm{m} / \mathrm{z}): 648\left(\mathrm{M}^{+}\right.$, $15.95 \%), 105$ (25.53\%), 77 (45.87\%), 50 (100\%).

\subsubsection{4-(2,4-Dichlorophenyl)-6-(4-(6-iodo-4-oxo-2-phenylquinazolin-3(4H)-yl)phenyl)-2- oxo-1,2-dihydropyridine-3-carbonitrile $\left(10_{b}\right)$}

Yield, $85 \%$; m.p.: $351-3^{\circ} \mathrm{C}$. Analysis for $\mathrm{C}_{32} \mathrm{H}_{17} \mathrm{Cl}_{2} \mathrm{IN}_{4} \mathrm{O}_{2}$ (m. w. 687); Calcd.: C, 55.92; H, 2.49; N, 8.15. Found: C, 55.98; H, 2.51; N, 8.19. IR (KBr, cm $\left.{ }^{-1}\right): 3339$ (OH enolic of pyridone), 3069 (CH aromatic), $2227(\mathrm{CN}), 1672\left(\mathrm{C}=\mathrm{O}\right.$ quinazolinone). $\mathrm{MS}(\mathrm{m} / \mathrm{z}): 688\left(\mathrm{M}^{+1}, 5.33 \%\right), 135$ (7.27\%), 105 (47.41\%), 77 (100\%), 50 (29.29\%).

\subsubsection{4-(2,6-Dichlorophenyl)-6-(4-(6-iodo-4-oxo-2-phenylquinazolin-3(4H)-yl)phenyl)-2- oxo-1,2-dihydropyridine-3-carbonitrile $\left(10_{c}\right)$}

Yield, $70 \%$; m.p.: $350-2^{\circ} \mathrm{C}$. Analysis for $\mathrm{C}_{32} \mathrm{H}_{17} \mathrm{Cl}_{2} \mathrm{IN}_{4} \mathrm{O}_{2}$ (m. w. 687); Calcd.: C, 55.92; H, 2.49; N, 8.15. Found: C, 55.96; H, 2.47; N, 8.19. IR (KBr, cm $\left.{ }^{-1}\right): 3339$ (OH enolic of pyridone), 3067 (CH aromatic), $2225(\mathrm{CN}), 1671\left(\mathrm{C}=\mathrm{O}\right.$ quinazolinone). ${ }^{1} \mathrm{HNMR}$ (DMSO- $\left.\mathrm{d}_{6}, \delta, \mathrm{ppm}\right): 7.53-8.52$ $(\mathrm{m}, 16 \mathrm{H}$, aromatic protons including $1 \mathrm{H}$ of pyridone $), 10.83(\mathrm{~s}, 1 \mathrm{H}, \mathrm{NH})\left(\mathrm{D}_{2} \mathrm{O}\right.$ exchangeable $)$, 
$11.31\left(\mathrm{~s}, 1 \mathrm{H}, \mathrm{OH}\right.$ of resonance) $\left(\mathrm{D}_{2} \mathrm{O}\right.$ exchangeable). $\mathrm{MS}(\mathrm{m} / \mathrm{z}): 687\left(\mathrm{M}^{+}, 0.4 \%\right), 350(14.80 \%)$, 135 (86.30\%), 105 (100\%), 77 (77.80\%), 50 (12.60\%).

\subsubsection{6-(4-(6-Iodo-4-oxo-2-phenylquinazolin-3(4H)-yl)phenyl)-2-oxo-4-(2,4,6-trimethoxy- phenyl)-1,2-dihydropyridine-3-carbonitrile $\left(10_{d}\right)$}

Yield, $75 \%$; m.p.: 383-5 ${ }^{\circ} \mathrm{C}$. Analysis for $\mathrm{C}_{35} \mathrm{H}_{25} \mathrm{IN}_{4} \mathrm{O}_{5}$ (m. w. 708); Calcd.: C, 59.33; H, 3.56; N, 7.91. Found: $\mathrm{C}, 59.37 ; \mathrm{H}, 3.58 ; \mathrm{N}, 7.98$. IR $\left(\mathrm{KBr}, \mathrm{cm}^{-1}\right): 3340(\mathrm{OH}$ enolic of pyridone), $3064(\mathrm{CH}$ aromatic), $2228(\mathrm{CN}), 1673\left(\mathrm{C}=\mathrm{O}\right.$ quinazolinone). ${ }^{1} \mathrm{HNMR}$ (DMSO-d $\left.\mathrm{d}_{6}, \delta, \mathrm{ppm}\right): 7.53-8.20(\mathrm{~m}$, $15 \mathrm{H}$, aromatic protons including $1 \mathrm{H}$ of pyridone), $3.27\left(\mathrm{~s}, 9 \mathrm{H}, 3 \mathrm{OCH}_{3}\right), 10.83(\mathrm{~s}, 1 \mathrm{H}, \mathrm{NH})\left(\mathrm{D}_{2} \mathrm{O}\right.$ exchangeable), 11.31 ( $\mathrm{s}, 1 \mathrm{H}, \mathrm{OH}$ of resonance) $\left(\mathrm{D}_{2} \mathrm{O}\right.$ exchangeable).

\subsubsection{6-(4-(6-Iodo-4-oxo-2-phenylquinazolin-3(4H)-yl)phenyl)-2-oxo-4-(thiophen-2-yl)-1,2- dihydropyridine-3-carbonitrile $\left(10_{e}\right)$}

Yield, $80 \%$; m.p.: 312-4 ${ }^{\circ} \mathrm{C}$. Analysis for $\mathrm{C}_{30} \mathrm{H}_{17} \mathrm{IN}_{4} \mathrm{O}_{2} \mathrm{~S}$ (m. w. 624); Calcd.: C, 57.70; H, 2.74; N, 8.97. Found: $\mathrm{C}, 57.78 ; \mathrm{H}, 2.76 ; \mathrm{N}, 9.04$. IR $\left(\mathrm{KBr}, \mathrm{cm}^{-1}\right): 3342$ (OH enolic of pyridone), 3037 (CH aromatic), $2234(\mathrm{CN}), 1674\left(\mathrm{C}=\mathrm{O}\right.$ quinazolinone). $\mathrm{MS}(\mathrm{m} / \mathrm{z}): 623\left(\mathrm{M}^{-1}, 0.57 \%\right), 351(47.63 \%)$, $135(100 \%), 105$ (70.19\%), 77 (67.33\%), 50 (24.61\%).

\subsubsection{2-Imino-6-(4-(6-iodo-4-oxo-2-phenylquinazolin-3(4H)-yl)phenyl)-4-substituted-1,2- dihydropyridine-3-carbonitrile (11 a-e)}

\section{General method:}

A mixture of ketone (4) $(0.93 \mathrm{~g}, 0.002 \mathrm{~mol})$, malononitrile $(0.12 \mathrm{ml}, 0.002 \mathrm{~mol})$, anhydrous ammonium acetate $(1.24 \mathrm{~g}, 0.016 \mathrm{~mol})$ and the appropriate aldehyde $(0.002 \mathrm{~mol})$ in $10 \mathrm{ml}$ of $\mathrm{n}$ butanol was refluxed for 5 hours. The reaction mixture was concentrated to half of its volume under reduced pressure. After cooling, the formed precipitate was filtered, air dried and crystallized from ethanol to afford the corresponding compounds $\left(\mathbf{1 1}_{\mathrm{a}-\mathrm{e}}\right)$ respectively.

\subsubsection{2-Imino-6-(4-(6-iodo-4-oxo-2-phenylquinazolin-3(4H)-yl)phenyl)-4-(4-methoxy- phenyl)-1,2-dihydropyridine-3-carbonitrile $\left(11_{a}\right)$}

Yield, $81 \%$; m.p.: $285-7^{\circ} \mathrm{C}$. Analysis for $\mathrm{C}_{33} \mathrm{H}_{22} \mathrm{IN}_{5} \mathrm{O}_{2}$ (m. w. 647); Calcd.: C, 61.22; H, 3.42; N, 10.82. Found: C, 61.21; H, 3.48; N, 10.88. IR ( $\left.\mathrm{KBr}, \mathrm{cm}^{-1}\right): 3331$ (2NH overlapped), $3037(\mathrm{CH}$ aromatic), $2220(\mathrm{CN}), 1672\left(\mathrm{C}=\mathrm{O}\right.$ quinazolinone). ${ }^{1} \mathrm{HNMR}$ (DMSO-d $\left.{ }_{6}, \delta, \mathrm{ppm}\right): 3.29(\mathrm{~s}, 3 \mathrm{H}$, $\left.\mathrm{OCH}_{3}\right), 7.53-8.20(\mathrm{~m}, 17 \mathrm{H}$, aromatic protons including $1 \mathrm{H}$ of pyridine $), 10.83(\mathrm{~s}, 1 \mathrm{H}, \mathrm{HN}), 11.31$ (s, 1H, NH=C). MS (m/z): $647\left(\mathrm{M}^{+}, 6.89 \%\right), 351$ (27.18\%), 135 (36.29\%), 105 (91.42\%), 77 (100 $\%), 63(34.47 \%), 51(47.98 \%)$.

\subsubsection{4-(2,4-Dichlorophenyl)-2-imino-6-(4-(6-iodo-4-oxo-2-phenylquinazolin-3(4H)-yl)- phenyl)-1,2-dihydropyridine-3-carbonitrile $\left(11_{b}\right)$}

Yield, $84 \%$; m.p.: $332-4^{\circ} \mathrm{C}$. Analysis for $\mathrm{C}_{32} \mathrm{H}_{18} \mathrm{Cl}_{2} \mathrm{IN}_{5} \mathrm{O}$ (m. w. 686); Calcd.: C, 56.00; H, 2.64; $\mathrm{N}, 10.20$. Found: C, 56.05; H, 2.67; N, 10.28. IR $\left(\mathrm{KBr}, \mathrm{cm}^{-1}\right): 3390,3282(2 \mathrm{NH}), 3076(\mathrm{CH}$ aromatic), $2228(\mathrm{CN}), 1683\left(\mathrm{C}=\mathrm{O}\right.$ quinazolinone). $\mathrm{MS}(\mathrm{m} / \mathrm{z}): 686\left(\mathrm{M}^{+}, 2.12 \%\right), 350(0.36 \%), 105$ (34.69\%), $76(100 \%), 50(46.07 \%)$.

\subsubsection{4-(2,6-Dichlorophenyl)-2-imino-6-(4-(6-iodo-4-oxo-2-phenylquinazolin-3(4H)-yl)- phenyl)-1,2-dihydropyridine-3-carbonitrile $\left(11_{c}\right)$}

Yield, $80 \%$; m.p.: 330-2 ${ }^{\circ} \mathrm{C}$. Analysis for $\mathrm{C}_{32} \mathrm{H}_{18} \mathrm{Cl}_{2} \mathrm{IN}_{5} \mathrm{O}$ (m. w. 686); Calcd.: C, 56.00; H, 2.64; $\mathrm{N}, 10.20$. Found: C, 56.06; H, 2.69; N, 10.29. IR $\left(\mathrm{KBr}, \mathrm{cm}^{-1}\right): 3309,3193(2 \mathrm{NH}), 3076(\mathrm{CH}$ aromatic), $2223(\mathrm{CN}), 1679\left(\mathrm{C}=\mathrm{O}\right.$ quinazolinone). MS (m/z): $685\left(\mathrm{M}^{-1}, 4.93 \%\right), 351(26.01 \%)$, $105(60.40 \%), 77(100 \%), 51(87.40 \%)$. 


\subsubsection{2-Imino-6-(4-(6-iodo-4-oxo-2-phenylquinazolin-3(4H)-yl)phenyl)-4-(3,4,5-trimethoxy- phenyl)-1,2-dihydropyridine-3-carbonitrile $\left(11_{d}\right)$}

Yield, $90 \%$; m.p.: $355-7^{\circ} \mathrm{C}$. Analysis for $\mathrm{C}_{35} \mathrm{H}_{26} \mathrm{IN}_{5} \mathrm{O}_{4}$ (m. w. 707); Calcd.: C, 59.42; H, 3.70; N, 9.90. Found: C, 59.48; H, 3.78; N, 9.95. IR $\left(\mathrm{KBr}, \mathrm{cm}^{-1}\right): 3336,3182(2 \mathrm{NH}), 3049$ (CH aromatic), $2222(\mathrm{CN}), 1671\left(\mathrm{C}=\mathrm{O}\right.$ quinazolinone). ${ }^{1} \mathrm{HNMR}\left(\mathrm{DMSO}_{6}, \delta, \mathrm{ppm}\right): 3.29\left(\mathrm{~s}, 9 \mathrm{H}, 3 \mathrm{OCH}_{3}\right), 7.56-$ $8.27(\mathrm{~m}, 15 \mathrm{H}$, aromatic protons including $1 \mathrm{H}$ of pyridine $), 10.83(\mathrm{~s}, 1 \mathrm{H}, \mathrm{HN}), 11.31(\mathrm{~s}, 1 \mathrm{H}$, $\mathrm{NH}=\mathrm{C}) . \mathrm{MS}(\mathrm{m} / \mathrm{z}): 707\left(\mathrm{M}^{+}, 0.04 \%\right), 351$ (81.17\%), 135 (85.49\%), 105 (83.91\%), 76 (100\%), 51 $(33.44 \%)$.

\subsubsection{2-Imino-6-(4-(6-iodo-4-oxo-2-phenylquinazolin-3(4H)-yl)phenyl)-4-(thiophen-2-yl)- 1,2-dihydropyridine-3-carbonitrile $\left(11_{e}\right)$}

Yield, $85 \%$; m.p.: 303-5 $5^{\circ}$. Analysis for $\mathrm{C}_{30} \mathrm{H}_{18} \mathrm{IN}_{5} \mathrm{OS}$ (m. w. 623); Calcd.: C, 57.79; H, 2.91; N, 11.23. Found: C, 57.83; H, 2.97; N, 11.32. IR $\left(\mathrm{KBr}, \mathrm{cm}^{-1}\right): 3334,3191(2 \mathrm{NH}), 3065(\mathrm{CH}$ aromatic), $2210(\mathrm{CN}), 1668\left(\mathrm{C}=\mathrm{O}\right.$ quinazolinone). $\mathrm{MS}(\mathrm{m} / \mathrm{z}): 623\left(\mathrm{M}^{+}, 0.86 \%\right), 236(3.44 \%), 105$ (16.66\%), 91 (23.26\%), 71 (58\%), 60 (100\%), 51 (37.18\%).

\subsubsection{2-Amino-6-(4-(6-iodo-4-oxo-2-phenylquinazolin-3(4H)-yl)phenyl)-4-substituted-4H- pyran-3-carbonitrile $\left(12_{a-d}\right)$}

\section{General method:}

A mixture of ketone (4) $(0.93 \mathrm{~g}, 0.002 \mathrm{~mol})$, malononitrile $(0.12 \mathrm{ml}, 0.002 \mathrm{~mol})$ and the appropriate aromatic aldehyde $(0.002 \mathrm{~mol})$ with few drops of piperidine in $10 \mathrm{ml}$ of $\mathrm{n}$-butanol was refluxed for 4 hours. The reaction mixture was concentrated to half of its volume under reduced pressure. After cooling, the formed precipitate was filtered, washed with cold water, air dried and crystallized from ethanol to give the corresponding compounds $\left(\mathbf{1 2}_{\mathrm{a}-\mathrm{d}}\right)$ respectively.

\subsubsection{2-Amino-4-(2,4-dichlorophenyl)-6-(4-(6-iodo-4-oxo-2-phenylquinazolin-3(4H)-yl)- phenyl)-4H-pyran-3-carbonitrile $\left(12_{a}\right)$}

Yield, $91 \%$; m.p.: 230-2 ${ }^{\circ} \mathrm{C}$. Analysis for $\mathrm{C}_{32} \mathrm{H}_{19} \mathrm{Cl}_{2} \mathrm{IN}_{4} \mathrm{O}_{2}$ (m. w. 688); Calcd.: C, 55.76; H, 2.78; N, 8.13. Found: C, 55.78; H, 2.83; N, 8.23. IR $\left(\mathrm{KBr}, \mathrm{cm}^{-1}\right): 3293\left(\mathrm{NH}_{2}\right), 3073$ (CH aromatic), $2191(\mathrm{CN}), 1667\left(\mathrm{C}=\mathrm{O}\right.$ of quinazolinone). MS (m/z): $688\left(\mathrm{M}^{+}, 0.07 \%\right), 351(29.21 \%), 135$ (82.55\%), 105 (94.49\%), 76 (100\%), 50 (28.39\%).

\subsubsection{2-Amino-4-(2,6-dichlorophenyl)-6-(4-(6-iodo-4-oxo-2-phenylquinazolin-3(4H)-yl)- phenyl)-4H-pyran-3-carbonitrile $\left(12_{b}\right)$}

Yield, $88 \%$; m.p.: $227-9^{\circ} \mathrm{C}$. Analysis for $\mathrm{C}_{32} \mathrm{H}_{19} \mathrm{Cl}_{2} \mathrm{IN}_{4} \mathrm{O}_{2}$ (m. w. 688); Calcd.: C, 55.76; H, 2.78; N, 8.13. Found: C, 55.81; H, 2.81; N, 8.18. IR $\left(\mathrm{KBr}, \mathrm{cm}^{-1}\right): 3307\left(\mathrm{NH}_{2}\right), 3083(\mathrm{CH}$ aromatic), $2193(\mathrm{CN}), 1668(\mathrm{C}=\mathrm{O}$ of quinazolinone $) . \mathrm{MS}(\mathrm{m} / \mathrm{z}): 688\left(\mathrm{M}^{+}, 0.11 \%\right), 351(51.02 \%), 135$ (76.20\%), 105 (78.56\%), $76(100 \%), 50(37.07 \%)$.

\subsubsection{2-Amino-6-(4-(6-iodo-4-oxo-2-phenylquinazolin-3(4H)-yl)phenyl)-4-(3,4,5-trimethoxy- phenyl)-4H-pyran-3-carbonitrile $\left(12_{c}\right)$}

Yield, $85 \%$; m.p.: $255-7^{\circ} \mathrm{C}$. Analysis for $\mathrm{C}_{35} \mathrm{H}_{27} \mathrm{IN}_{4} \mathrm{O}_{5}$ (m. w. 710); Calcd.: C, 59.16; H, 3.83; N, 7.89. Found: C, 59.20; H, 3.86; N, 8.02. IR (KBr, cm $\left.{ }^{-1}\right): 3340\left(\mathrm{NH}_{2}\right), 3064(\mathrm{CH}$ aromatic), 2191 $(\mathrm{CN}), 1668\left(\mathrm{C}=\mathrm{O}\right.$ of quinazolinone). MS (m/z): $709\left(\mathrm{M}^{-1}, 0.06 \%\right), 350(89.32 \%), 135$ (33.07\%), $105(99.87 \%), 77(100 \%), 51(57.69 \%)$.

\subsubsection{2-Amino-6-(4-(6-iodo-4-oxo-2-phenylquinazolin-3(4H)-yl)phenyl)-4-(thiophen-2-yl)- 4H-pyran-3-carbonitrile $\left(12_{d}\right)$}

Yield, $84 \%$; m.p.: 190-2 ${ }^{\circ} \mathrm{C}$. Analysis for $\mathrm{C}_{30} \mathrm{H}_{19} \mathrm{IN}_{4} \mathrm{O}_{2} \mathrm{~S}$ (m. w. 626); Calcd.: C, 57.52; H, 3.06; N, 8.94. Found: C, 57.58; H, 3.05; N, 9.06. IR (KBr, cm $\left.{ }^{-1}\right): 3332\left(\mathrm{NH}_{2}\right), 3089$ (CH aromatic), 2187 
$(\mathrm{CN}), 1662\left(\mathrm{C}=\mathrm{O}\right.$ of quinazolinone). MS (m/z): $625\left(\mathrm{M}^{-1}, 0.15 \%\right), 350(24.99 \%), 135(19.99 \%)$, $105(100 \%), 77(44.25 \%), 63(19.53 \%)$.

\section{Docking studies}

In the present work, all the target compounds were subjected to docking study to explore their binding mode to COX-2 enzyme. All modeling experiments were performed using molsoft (ICM-Pro) program which provides a unique set of tools for the modeling of protein / ligand interactions. It predicts how small flexible molecule such as substrates or drug candidates bind to a protein of known 3D structure represented by grid interaction potentials (file:///D:/molsoft\%20from\%20net-/molsoft\%20from\%20net/icm_pro.html). Each experiment used the biological target COX-2 downloaded from the Brookhaven Protein Databank (www. rcsb. Org PDB ID 1PXX). In order to qualify the docking results in terms of accuracy of the predicted binding conformations in comparison with the experimental procedure, the reported COX-2 inhibitor drug (Indomethacin) was used as a reference ligand. The docking study has been conducted to predict the binding mode and to rationalize the observed biological activity.

\section{Biological evaluation}

\section{a. Anti-inflammatory screening}

The animal studies were undertaken with approval from the Ethics Committee (approval \# 23PD/3/12/8R) of Al-Azhar University, Nasr City, Cairo, Egypt. All the trials were carried out according to the respective internationally guidelines. Adult rats of both sexes weighing 150-200 g were used as experimental animals. They were obtained from an animal facility (Animal house, Department of Pharmacology and Toxicology, Faculty of Pharmacy, Al-Azhar University). Animals were housed in stainless steel wire-floored cages without any stressful stimuli. Animals were kept under well-ventilated conditions at room temperature $\left(25-30^{\circ} \mathrm{C}\right)$. They were fed on an adequate standard laboratory chow (El-Nasr Co., Abou-Zabal, Egypt) and allowed to acclimatize with free access to food and water for 24 hours period before testing except during the short time they were removed from the cages for testing. The animals were randomly arranged in twelve groups, each of six animals, each kept in a separate cage. Indomethacin (Khahira Pharmaceutical and Chemical Co., Cairo, Egypt) was used as a reference drug. Carrageenan (Sigma-Aldrich Chemical Co., USA) was used to induce inflammation in the experimental animals.

The carrageenan rat paw oedema model of inflammation was used to evaluate the antiinflammatory properties of the tested compounds (Winter et al., 1962). Groups of six rats were administered the graded doses of the test compounds or indomethacin $(10 \mathrm{mg} / \mathrm{kg} \mathrm{b}$ wt in $1 \mathrm{ml}$ saline) orally. Control animals received an equal volume of saline $(1 \mathrm{ml})$. After one hour, sterile carrageenan $(0.1 \mathrm{ml}$ of a $1 \% \mathrm{w} / \mathrm{v}$ solution in saline) was injected sub-planter into right hind paw of the rat. The contralateral hind paw received the same volume of saline and served as a normal control. Carrageenan caused visible redness and pronounced swelling that was well developed by $4 \mathrm{~h}$ and persisted for more than $48 \mathrm{~h}$. Right hind paw was measured with a micrometer caliber (Meng et al., 1999) before, and at 1, 2, 3 and $4 \mathrm{~h}$ after carrageenan injection.

The anti-inflammatory activity was expressed as percentage inhibition of edema volume in the treated animals in comparison with the control group. \% Inhibition of edema $=\left(\mathrm{V}_{\mathrm{c}}-\mathrm{V}_{\mathrm{t}}\right)(100) /$ $\mathrm{V}_{\mathrm{c}}$ where $\mathrm{V}_{\mathrm{c}}$ and $\mathrm{V}_{\mathrm{t}}$ are the volumes of edema for the control and tested substance-treated animal groups, respectively, while potency of the tested compounds was calculated regarding indomethacin, reference standard, treated group according to the following equation: \% Potency $=$ (\%Edema inhibition of tested compound treated group) (100) / \%Edema inhibition of indomethacin treated group. 


\section{b. Gastric ulcerogenic studies}

Groups of 5 male rats with a weight between 150 and $175 \mathrm{~g}$ are used. They are starved $24 \mathrm{~h}$ prior to drug administration. The test compounds or indomethacin were administered orally in 10 $\mathrm{mg} / \mathrm{kg} \mathrm{b}$ wt as aqueous suspension. Control group received ethanol (1 $\mathrm{ml}$ of $100 \%)$ orally. The animals are sacrificed after $7 \mathrm{~h}$. Stomachs are removed and placed on saline soaked filter paper until inspection. A longitudinal incision along the greater curvature is made with fine scissor. The stomach is rinsed with saline, extended on a plastic board and the presence or the absence of gastric irritation is determined. The presence of a single or multiple lesions (erosion, ulcer or perforation) is considered to be positive (Amr and Abdulla 2006). The number and severity of mucosal lesions were noted and lesions were scaled as follows: petechial lesions $=1$, lesions less than $1 \mathrm{~mm}=2$, lesion between 1 and $2 \mathrm{~mm}=3$, lesions between 2 and $4 \mathrm{~mm}=4$, lesions more than $4 \mathrm{~mm}=5$. A total lesion score for each animal is calculated as the total number of lesions multiplied by the respective severity scores and the results are expressed as the severity of lesions / rat (Mozsik et al., 1985).

\section{CONCLUSION: -}

This study includes the synthesis of novel series of 6-iodo-2-phenyl-quinazolin-4(3H)-ones attached to various heterocyclic ring systems such as: pyrazoline, pyrimidin-2-one, pyrimidin-2thione, 2-oxo(imino)pyridine and pyran at 3rd position to obtain safer and potent antiinflammatory agents. All the newly synthesized quinazoline derivatives were docked into the same pocket of indomethacin in COX-2 and have shown good docking results and good fitting into the active site. Different ten derivatives were evaluated as anti-inflammatory agents in experimental animals. The highest binding affinities were noticed for compounds $\mathbf{8}_{\mathbf{a}}, \mathbf{1 2}_{\mathbf{b}}$ and $\mathbf{1 0}_{\mathbf{b}}$ which showed the highest anti-inflammatory activities with superior gastrointestinal safety profile when compared to indomethacin. It has been found that compound $\mathbf{8}_{\mathbf{a}}$ showed the highest antiinflammatory activity and compound $\mathbf{1 2}_{\mathbf{b}}$ exhibited the least ulcerogenic effect in all of the experimental animals. Thus, it can be concluded that pyrimidin-2-one, pyran and pyridone ring systems substituted with highly lipophilic moieties are important for anti-inflammatory activity of potent safety margin profiles towards G.I.T. By the use of molecular modeling we realized the mechanism of their effects that could be their interactions with the same residues that interact with indomethacin.

ACKNOLEDGMENT: - The authors express deep thanks to Prof. Dr. Ahmed M. Mansour, Pharmacology \& Toxicology Department, Faculty of Pharmacy (Boys' branch), AlAzhar University, Nasr City, Cairo, Egypt, for carrying out the pharmacological screening.

\section{REFERENCES}

Abbas, S. E., Abdel Gawad, N. M., Georgey, H. H. and Abdullah, J. H.; (2010): Int. J. ChemTech. Res., 2 (3), 1560-1578.

Abou-Seri, S. M., Abouzid, K. and Abou El Ella, D. A.; (2011): Eur. J. Med. Chem. 46(2), 647658.

Alafeefy, A. M., Kadi, A. A., Al-Deeb, O. A., El-Tahir, K. E. H. and Al-jaber, N. A.; (2010): Eur. J. Med. Chem. 45 (11), 4947-4952.

Alagarsamy, V., Kavitha, K., Rupeshkumar, M., Solomon, V. R., Kumar, J., Kumar, D. S. and Sharma, H. K.; (2009): Acta Pharm. 59(1), 97-106.

Amin, K. M., Kamel, M. M., Anwar, M. M., Khedr, M. and Syam, Y.M.; (2010): Eur. J. Med. Chem. 45, 2117-2131.

Amr, A. E. and Abdulla, M. M.; (2006): Arch. Pharm. Chem. Life Sci. 339, 88-95.

Chakraborti, A. K. and Thilagavathi, R; (2003): Bioorg. Med. Chem. 11, 3989-3996. 
Chandrika, P. M., Rao, A. R. R., Narsaiah, B. and Raju, M. B.; (2008): Int. J. Chem. Sci. $\underline{6}(3)$, 1119-1146.

Hassanzadeh, F., Jafari, E., Hakimelahi, G. H., Khajouei1, M. R., Jalali, M. and Khodarahmi, G.A.; (2012): Res. Pharm. Sci. Z(2), 87-94.

Krishnan, S. K., Ganguly, S., Veerasamy, R. and Jan, B.; (2011): Eur. Rev. Med. Pharmacol. Sci. 15(6), 673-681.

Kumar, P., Shrivastava, B., Pandeya, S. N. and Stables, J. P.; (2011): Eur. J. Med. Chem. 46, 1006-1018.

Meng, L., Mohan, R., Kwok, B. H. B., Elofssof, M., Sin, N. and Crews, C. M.; (1999): Proc. Natl. Acad. Sci. USA, 96, 10403-10408.

Mohamed, M. S., Kamel, M. M., Kassem, E. M. M., Abotaleb, N., Khedr, M. and Ahmed, M. F.; (2011): Acta Polo. Pharm. Drug Res. 68(5), 665-675.

Mohamed, M. S., Kamel, M. M., Kassem, E. M. M., Abotaleb, N., Nofal, S. M. and Ahmed, M. F.; (2010): Acta Polo. Pharm. Drug Res. 67(2), 159-171.

Mohamed, M. S., Kamel, M. M., Kassem, E. M. M., Abotaleb, N., Nofal, S. M. and Ahmed, M. F.; (2009): Acta Polo. Pharm. Drug Res. 66 (5), 487-500.

Mozsik G.Y., Moron F. and Javor T.; (1985): Leukot. Med. 9, 71.

Myangar, K. N. and Raval, J. P.; (2012): Med. Chem. Res. 21(10), 2762-2771.

Naganaboina, V. K., Chandra, K. L, Desper, J. and Rayat, S.; (2011): Org. Lett. 13, 3718-3721.

Patil, A., Ganguly, S. and Surana, S.; (2010): J. Chem. Sci. 122, 443-450.

Refaie, F. M., Esmat, A. Y., Abdel Gawad, S. M., Ibrahim, A. M. and Mohamed, M. A.; (2005): Lipids in Health and Dis. 4(22), 1-11.

Rajasekaran, S. and Gopalkrishna, R.; (2012): Int. J. ChemTech Res. 4(3), 1207-1211.

Rajveer, C. H., Kumaraswamy, D., Sudharshini, S. and Rathinaraj, B. S.; (2010): Int. J. Pharma Bio Sci. 1 (3), 1-10.

Sati, N., Kumar, S. and Rawat, M. S. M.; (2009): Indian J. Pharm. Sci. 71(5), 572-575.

Sen, D., Banerjee, A., Ghosh, A. K. and Chatterjee, T. K.; (2010): J. Adv. Pharm. Tech. Res. 1(4), 401-405.

Sirisoma, N., Pervin, A., Zhang, H., Jiang, S., Willardsen, J. A., Anderson, M. B., Mather, G., Pleiman, C. M., Kasibhatla, S., Tseng, B., Drewe, J. and Cai, S. X.; (2010): Bioorg. Med. Chem. Lett. 20(7), 2330-2334.

Winter, G.A., Rislfy, E. A. and Nuss, G.W.; (1962): Proc. Soc. Exp. Biol. Med. 111, 544.

تصميم و تشييد وتقييم حيوى ونمذجه جزيئيه لبعض المشتثات الجديدة من نواة كينازولين- ع -ون

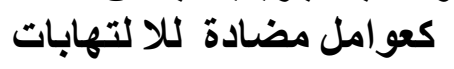

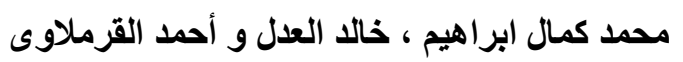

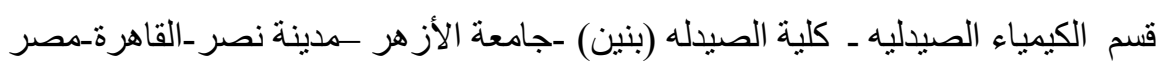

تم فى هذا البحث تصميم و تشييد بعض المركبات الجديدة من نواة الكينازولين وقد نم اثبات التركيب البنائى لهذه الهئ

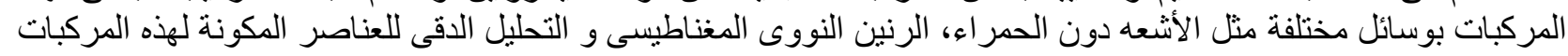

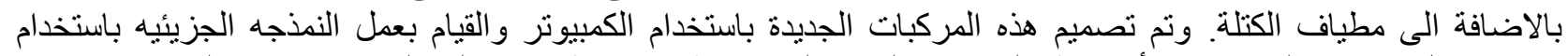

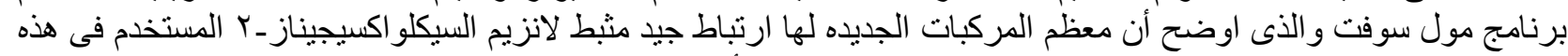

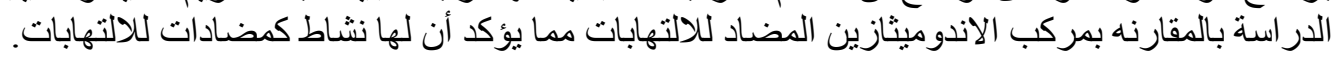

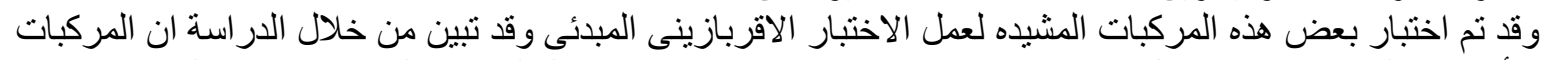

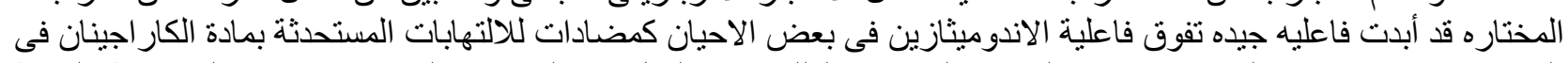

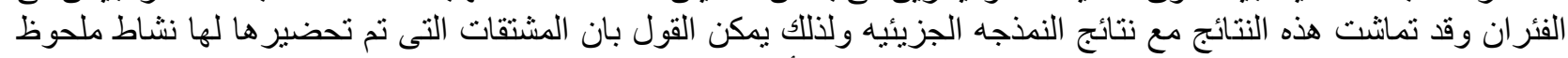

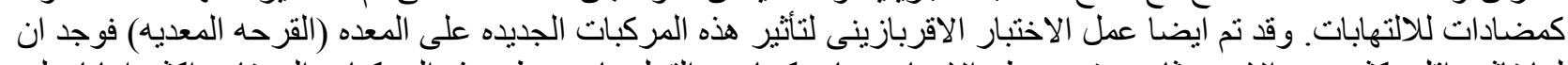

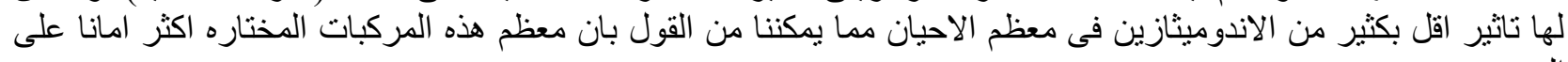

